Interfaces and Free Boundaries 15 (2013), 203-236

DOI $10.4171 / \mathrm{IFB} / 301$

\title{
Scalar conservation laws on moving hypersurfaces
}

\author{
GERHARD DZIUK \\ Abteilung für Angewandte Mathematik, University of Freiburg, Hermann-Herder-Straße 10, 79104 \\ Freiburg i. Br., Germany \\ E-mail: gerd.dziuk@mathematik.uni-freiburg.de \\ DIETMAR KRÖNER \\ Abteilung für Angewandte Mathematik, University of Freiburg, Hermann-Herder-Straße 10, 79104 \\ Freiburg i. Br., Germany \\ E-mail:dietmar@mathematik.uni-freiburg.de \\ THOMAS MÜLLER \\ Abteilung für Angewandte Mathematik, University of Freiburg, Hermann-Herder-Straße 10, 79104 \\ Freiburg i. Br., Germany \\ E-mail:mueller@mathematik.uni-freiburg.de
}

[Received 5 July 2012 and in revised form 5 April 2013]

\begin{abstract}
We consider conservation laws on moving hypersurfaces. In this work the velocity of the surface is prescribed. But one may think of the velocity to be given by PDEs in the bulk phase. We prove existence and uniqueness for a scalar conservation law on the moving surface. This is done via a parabolic regularization of the hyperbolic PDE. We then prove suitable estimates for the solution of the regularized PDE, that are independent of the regularization parameter. We introduce the concept of an entropy solution for a scalar conservation law on a moving hypersurface. We also present some numerical experiments. As in the Euclidean case we expect discontinuous solutions, in particular shocks. It turns out that in addition to the "Euclidean shocks" geometrically induced shocks may appear.
\end{abstract}

2010 Mathematics Subject Classification: Primary 35L65, 58J45, 76N10, 65M08.

Keywords: Hyperbolic; Conservation Laws; Evolving Surfaces; Total Variation Estimates; Finite Volume Schemes.

\section{Introduction}

The theoretical and numerical solution of partial differential equations on stationary or moving surfaces has become quite important during the last decade. In many applications PDEs in bulk phases are coupled to PDEs on interfaces between these phases. The modeling of transport processes for quantities on evolving fluid interfaces with surrounding bulk phases has already been established in [9] and the references therein. There is a satisfactory analysis and numerical analysis for elliptic and parabolic equations on stationary or moving surfaces. For references we refer to [14], [15], [16]. Several phenomena like shallow water equations on the earth, relativistic flows, transport processes on surfaces, transport of oil on the waves of the ocean or the transport on moving interfaces between two fluids are modeled by transport equations, and thus hyperbolic PDEs, on fixed or moving surfaces. These equations often are highly nonlinear. 
In this work we study scalar conservation laws on moving hypersurfaces without boundary in $\mathbb{R}^{n+1}$. The motion of the surface is prescribed. Assume that $\Gamma(t)$ is a family of smooth and compact hypersurfaces which moves smoothly with time $t \in[0, T]$. When the scalar material quantity $u=$ $u(x, t), x \in \Gamma(t), t \in[0, T]$, is propagated with the surface and simultaneously transported via a given flux $f=f((x, t), u)$ on the surface, then its evolution with respect to prescribed initial values $u_{0}$ is governed by the initial value problem

$$
\dot{u}+u \nabla_{\Gamma} \cdot v+\nabla_{\Gamma} \cdot(f(\cdot, u))=0 \quad \text { on } G_{T}, \quad u(\cdot, 0)=u_{0} \text { on } \Gamma_{0} .
$$

Here $v$ denotes the velocity of the surface $\Gamma$, and $\nabla_{\Gamma}$ is the surface gradient. The dot stands for a material derivative, $f$ is the given flux function which we assume to be tangentially divergence free on $\Gamma$ and which is a tangent vector to the surface. By $G_{T}$ we denote the space time surface

$$
G_{T}=\bigcup_{t \in(0, T)} \Gamma(t) \times\{t\} .
$$

The quantities appearing in the PDE (1.1) are well defined for $u: G_{T} \rightarrow \mathbb{R}$ and do not depend on the ambient space.

Let us briefly summarize the published results related to this topic. The study of scalar conservation laws on time independent manifolds was initiated by Panov [33]. The PDE considered in his paper is independent of the geometry which allows him to reduce the whole problem to the Euclidean case in order to prove existence and uniqueness. Total variation estimates for time independent Riemannian manifolds can be found in [22]. The existence proof of entropy solutions on time independent Riemannian manifolds is considered in [7] by viscous approximation. The ideas are based on Kruzkov's and DiPerna's theories for the Euclidean case. In a forthcoming paper Lengeler and one of the authors [28] are generalizing the results which we are going to prove in this contribution to the case of time dependent Riemannian manifolds. They show existence and uniqueness (in the space of measure-valued entropy solutions) of entropy solutions for initial values in $L^{\infty}$ and derive total variation estimates if the initial values are in BV. Additionally, initial boundary value problems for this type of PDE seem to be very interesting from the physical point of view and will be considered in future work. Convergence of finite volume schemes on time independent Riemannian manifolds can be found in [1]. In the paper [27] LeFloch, Okutmustur and Neves prove an error estimate of the form $\left\|u-u_{h}\right\|_{L^{1}} \leqslant c h^{\frac{1}{4}}$ for the scheme in [1]. The proof generalizes the ideas of the Euclidian case and the convergence rate is the same. This result was generalized to the time dependent case by Giesselmann [18] under the assumption that an entropy solution exists, which we are going to prove in this paper. In [2] an error estimate for hyperbolic conservation laws on an $(N+1)$ dimensional manifold (spacetime), whose flux is a field of differential forms of degree $N$, is shown. The matter Einstein equation for perfect fluids on spacetimes in the context of general relativity is considered in [26]. A wave propagation algorithm for hyperbolic systems on curved manifolds with application in relativistic hydrodynamics and magnetohydrodynamics have been developed and tested in [32], [4], [5], and a finite volume scheme on spherical domains, partially with adaptive grid refinement in [8], [5].

This paper is organized as follows. In Section 2 we will summarize the notations and basic relations for moving hypersurfaces which we need to show existence for (1.1). The PDE in (1.1) will be derived in Section 3. Since the weak solution of (1.1) is in general not unique we will define entropy solutions in Section 4. The idea for the existence proof is based on the approximation of the solution of (1.1) by the solution of a parabolic regularization, which will be presented in Section 5. 
In Section 6 and 7 we prove uniform estimates in the $H^{1,1}$-norm of the solution of the parabolic regularization. This implies compactness in $L^{1}$ and therefore existence for (1.1), which is the main subject of Section 8. Since this existence result depends on the special regularization, defined in Section 5, we have to prove uniqueness of (1.1) in Section 9. With the numerical algorithm described in Section 10 we have performed some numerical experiments. The results are shown in Section 11.

\section{Notations and basic relations for moving hypersurfaces}

In this section we present the description of the moving geometry. We use the notion of tangential or surface gradients.

Assumptions 2.1 Let $\Gamma_{t}=\Gamma(t) \subset \mathbb{R}^{n+1}$ for $t \in[0, T]$ be a time dependent, closed, smooth hypersurface. The initial surface $\Gamma_{0}$ is transported by the smooth function

$$
\Phi: \Gamma_{0} \times[0, T] \rightarrow \mathbb{R}^{n+1}
$$

with $\Phi\left(\Gamma_{0}, t\right)=\Gamma_{t}$ and $\Phi(\cdot, 0)=I d$. We assume that $\Phi(\cdot, t): \Gamma_{0} \rightarrow \Gamma(t)$ is a diffeomorphism for every $t \in[0, T]$. The velocity of the material points is denoted by $v:=\partial_{t} \Phi \circ \Phi^{-1}$. The tangential flow of a conservative material quantity $u$ with $u(\cdot, t): \Gamma_{t} \rightarrow \mathbb{R}$ is described by a flux function $f=f((x, t), u)$ which is a family of vector fields such that $f((x, t), u)$ is a tangent vector at the surface $\Gamma_{t}$ for $x \in \Gamma_{t}, t \in[0, T]$ and $u \in \mathbb{R}$. We assume that all derivatives of $\mathrm{f}$ are bounded and that $\nabla_{\Gamma} \cdot f((\cdot, t), s)=0$ for all fixed $t \in \mathbb{R}^{+}, s \in \mathbb{R}$. The definition of $\nabla_{\Gamma} \cdot$ is given below.

\subsection{Tangential derivatives and geometry}

Let us assume that $\Gamma$ is a compact $C^{2}$-hypersurface in $\mathbb{R}^{n+1}$ with normal vector field $v$.

DEFinition 2.2 For a differentiable function $g: \Gamma \rightarrow \mathbb{R}$ we define its tangential gradient as

$$
\nabla_{\Gamma} g=\nabla \bar{g}-\nabla \bar{g} \cdot v v
$$

where $\bar{g}$ is an extension of $g$ to a neighborhood of $\Gamma$. We denote the components of the gradient by

$$
\nabla_{\Gamma} g=\left(\underline{D}_{1} g, \ldots, \underline{D}_{n+1} g\right) .
$$

The Laplace-Beltrami operator then is given by

$$
\Delta_{\Gamma} g=\nabla_{\Gamma} \cdot \nabla_{\Gamma} g=\sum_{j=1}^{n+1} \underline{D}_{j} \underline{D}_{j} g .
$$

It is well known that the tangential gradient only depends on the values of $g$ on $\Gamma$. For more information about this concept we refer to [13]. With the help of tangential gradients we can describe the geometric properties of $\Gamma$. The matrix

$$
\mathcal{H}=\nabla_{\Gamma} v, \quad \mathcal{H}_{i j}=\left(\nabla_{\Gamma} v\right)_{i j}=\underline{D}_{i} v_{j}=\underline{D}_{j} v_{i} \quad(i, j=1, \ldots, n+1)
$$

has a zero eigenvalue in normal direction: $\mathcal{H} v=0$. The remaining eigenvalues $\kappa_{1}, \ldots, \kappa_{n}$ are the principal curvatures of $\Gamma$. We can view the matrix $\mathcal{H}$ as an extended Weingarten map. The mean 
curvature $H$ of $\Gamma$ is given as the trace of $\mathcal{H}$,

$$
H=\sum_{j=1}^{n+1} \varkappa_{j j}=\sum_{j=1}^{n} \kappa_{j},
$$

where we note, that this definition of the mean curvature differs from the common definition by a factor $\frac{1}{n}$. Integration by parts on a hypersurface $\Gamma$ is given by the following formula. A proof for surfaces without boundary can be found in [19]. The extension to surfaces with boundary is easily obtained. By $\mu$ we denote the conormal to $\partial \Gamma$.

LEMMA 2.3

$$
\int_{\Gamma} \nabla_{\Gamma} g=\int_{\Gamma} g H v+\int_{\partial \Gamma} g \mu
$$

Higher order tangential derivatives do not commute. But we have the following law for second derivatives. Here and in the following we use the summation convention that we sum over doubly appearing indices.

LEMmA 2.4 For a function $g \in C^{2}(\Gamma)$ we have for $i, k=1, \ldots, n+1$, that

$$
\underline{D}_{i} \underline{D}_{k} g=\underline{D}_{k} \underline{D}_{i} g+H_{k l} \underline{D}_{l} g v_{i}-\mathcal{H}_{i l} \underline{D}_{l} g v_{k} .
$$

For the convenience of the reader we give a short proof for this relation.

Proof. We use the definition (2.2) of the tangential gradient and extend $g$ constantly in normal direction to obtain $\bar{g}$. Then

$$
\begin{aligned}
\underline{D}_{i} \underline{D}_{k} g-\underline{D}_{k} \underline{D}_{i} g= & \underline{D}_{i}\left(\bar{g}_{x_{k}}-\bar{g}_{x_{l}} v_{l} v_{k}\right)-\underline{D}_{k}\left(\bar{g}_{x_{i}}-\bar{g}_{x_{m}} v_{m} v_{i}\right) \\
= & \bar{g}_{x_{k} x_{i}}-\bar{g}_{x_{k} x_{r}} v_{r} v_{i}-\left(\bar{g}_{x_{l} x_{i}}-\bar{g}_{x_{l} x_{s}} v_{s} v_{i}\right) v_{l} v_{k}-\bar{g}_{x_{l}}\left(\mathcal{H}_{i l} v_{k}+v_{l} \mathcal{H}_{i k}\right) \\
& \quad-\bar{g}_{x_{i} x_{k}}+\bar{g}_{x_{i} x_{r}} v_{r} v_{k}+\left(\bar{g}_{x_{m} x_{k}}-\bar{g}_{x_{m} x_{s}} v_{s} v_{k}\right) v_{m} v_{i} \\
\quad & \quad \bar{g}_{x_{m}}\left(\mathcal{H}_{m k} v_{i}+v_{m} \mathcal{H}_{i k}\right) \\
= & \bar{g}_{x_{m}} \mathcal{H}_{m k} v_{i}-\bar{g}_{x_{l}} \mathcal{H}_{i l} v_{k}=\mathcal{H}_{k l} v_{i} \underline{D}_{l} g-\mathcal{H}_{i l} v_{k} \underline{D}_{l} g .
\end{aligned}
$$

In the last step we have used that $\mathcal{H} v=0$.

\subsection{Material derivatives}

In this section we work with moving surfaces.

DEFINITION 2.5 For a differentiable function $g: G_{T} \rightarrow \mathbb{R}$ we define the material derivative

$$
\dot{g}=\frac{\partial g}{\partial t}+v \cdot \nabla g .
$$

Note that the material derivative only depends on the values of $g$ on the space-time surface $G_{T}$. In our proofs we will frequently use the following formula for the commutation of spatial (tangential) derivatives and (material) time derivatives. A proof is given in the Appendix. 
Lemma 2.6 For $g \in C^{2}\left(G_{T}\right)$ we have that

$$
\left(\underline{D}_{l} g\right)^{\cdot}=\underline{D}_{l} \dot{g}-A_{l r}(v) \underline{D}_{r} g
$$

with the matrix

$$
A_{l r}(v)=\underline{D}_{l} v_{r}-v_{s} v_{l} \underline{D}_{r} v_{s} \quad(l, r=1, \ldots, n+1) .
$$

\section{Derivation of the PDE}

We derive the conservation law, which we are going to solve in this paper. To this end we need the following transport theorem on moving surfaces respectively on portions $\gamma(t)$ of $\Gamma(t)$ which move in time according to the prescribed velocity $v$. A proof can be found in [14].

LEMMA 3.1

$$
\frac{d}{d t} \int_{\gamma(t)} g=\int_{\gamma(t)} \dot{g}+g \nabla_{\Gamma} \cdot v
$$

Let $u(\cdot, t)$ be a scalar quantity, defined on $\Gamma(t)$, which is conserved. The conservation law which we are going to solve is given in integral form by

$$
\frac{d}{d t} \int_{\gamma(t)} u=\int_{\partial \gamma(t)} Q \cdot \mu
$$

Here, $Q$ is a flux, which we will parametrize later. Obviously normal parts of $Q$ do not enter the conservation law, because the conormal $\mu$ on $\partial \gamma$ is a tangent vector. Thus we may assume that $Q$ is a tangent vector to $\Gamma$. But note, that even if we choose $Q$ as a vector with a normal part, then $Q \cdot \mu=P Q \cdot \mu$ with the projection $P_{i j}=\delta_{i j}-v_{i} v_{j}(i, j=1, \ldots, n+1)$. We apply integration by parts (2.3) to the right hand side of (3.2),

$$
\int_{\partial \gamma(t)} Q \cdot \mu=\int_{\gamma(t)} \nabla_{\Gamma} \cdot Q-\int_{\gamma(t)} H Q \cdot v=\int_{\gamma(t)} \nabla_{\Gamma} \cdot Q .
$$

To the left hand side of (3.2) we apply the transport theorem from Lemma 3.1. This leads to

$$
\frac{d}{d t} \int_{\gamma(t)} u=\int_{\gamma(t)} \dot{u}+u \nabla_{\Gamma} \cdot v
$$

Thus the equation (3.2) is equivalent to

$$
\int_{\gamma(t)} \dot{u}+u \nabla_{\Gamma} \cdot v-\nabla_{\Gamma} \cdot Q=0
$$

and since $\gamma$ is an arbitrary subregion of $\Gamma$, we arrive at the PDE

$$
\dot{u}+u \nabla_{\Gamma} \cdot v-\nabla_{\Gamma} \cdot Q=0 .
$$

Throughout this paper we assume, that $Q$ has the form

$$
Q=-f((x, t), u) \quad(x \in \Gamma(t)),
$$


where we assume that

$$
f(\cdot, u) \cdot v=0
$$

for all $u \in \mathbb{R}$ on $\Gamma$. With this parametrization of the flux the PDE (3.3) reads

$$
\dot{u}+u \nabla_{\Gamma} \cdot v+\nabla_{\Gamma} \cdot f(\cdot, u)=0 \quad \text { on } G_{T},
$$

where here, and in the following, by the divergence of $f$ we mean the 'total' divergence

$$
\begin{aligned}
\nabla_{\Gamma} \cdot f(\cdot, u) & =\sum_{j=1}^{n+1} \underline{D}_{j} f_{j}(\cdot, u)+\sum_{j=1}^{n+1} \frac{\partial f_{j}}{\partial u}(\cdot, u) \underline{D}_{j} u \\
& =\left(\nabla_{\Gamma} \cdot f\right)(\cdot, u)+f_{u}(\cdot, u) \cdot \nabla_{\Gamma} u
\end{aligned}
$$

REMARK 3.2 Note, that because of the condition (3.4) it is in general not possible to choose the flux $f$ independently of $x$ and $t$. If we start with a flux of the form $Q=\tilde{f}(u)$ in the law (3.2), then the PDE changes to

$$
\dot{u}+u \nabla_{\Gamma} \cdot v+\nabla_{\Gamma} \cdot P \tilde{f}(u)=0
$$

and we have $f((x, t), u)=P(x, t) \tilde{f}(u)$ in (3.5).

\section{Definition of entropy solutions}

As in the Euclidean case classical solutions of (1.1) do not exist globally in time in general. Therefore we have to introduce the notion of a weak solution.

Definition 4.1 A function $u \in L^{\infty}\left(G_{T}\right)$ is called a weak solution of (1.1) if

$$
\int_{0}^{T} \int_{\Gamma} u \dot{\varphi}+f(\cdot, u) \cdot \nabla_{\Gamma} \varphi+\int_{\Gamma_{0}} u_{0} \varphi(\cdot, 0)=0
$$

for all test functions $\varphi \in C^{1}\left(\overline{G_{T}}\right)$ with $\varphi(\cdot, T)=0$.

In general weak solutions are not unique. Therefore we select the entropy solution which will be introduced in Definition 4.3. For the motivation of the entropy condition given in (4.4), let us consider the following Lemma. Here and in the following we assume that $u_{0 \varepsilon} \in H^{2,1}\left(\Gamma_{0}\right) \cap L^{\infty}\left(\Gamma_{0}\right)$ and

$$
\left\|u_{0 \varepsilon}\right\|_{L^{\infty}\left(\Gamma_{0}\right)}+\left\|\nabla_{\Gamma_{0}} u_{0 \varepsilon}\right\|_{L^{1}\left(\Gamma_{0}\right)}+\varepsilon\left\|\nabla_{\Gamma_{0}}^{2} u_{0 \varepsilon}\right\|_{L^{1}\left(\Gamma_{0}\right)} \leqslant c_{0}
$$

with a constant $c_{0}$ which is independent of the parameter $0<\varepsilon \leqslant 1$, where the Sobolev space is given by

$$
H^{2,1}\left(\Gamma_{0}\right)=\left\{\eta \in L^{1}\left(\Gamma_{0}\right) \mid \nabla_{\Gamma_{0}} \eta \in L^{1}\left(\Gamma_{0}\right)^{n+1}, \nabla_{\Gamma_{0}}^{2} \eta \in L^{1}\left(\Gamma_{0}\right)^{(n+1) \times(n+1)}\right\} .
$$

LEMma 4.2 Let $f=\left(f_{1}, \ldots, f_{n+1}\right), q=\left(q_{1}, \ldots, q_{n+1}\right), \eta \in C^{2}(\mathbb{R}), \eta^{\prime \prime} \geqslant 0$. Define $q_{l}(\cdot, s):=$ $\int_{s_{0}}^{s} \eta^{\prime}(\tau) f_{l u}(\cdot, \tau) d \tau$ for $l=1, \ldots, n+1$ and let $u_{0} \in L^{\infty}\left(\Gamma_{0}\right)$. Assume that $u_{\varepsilon}$ is a smooth solution of

$$
\dot{u}_{\varepsilon}+u_{\varepsilon} \nabla_{\Gamma} \cdot v+\nabla_{\Gamma} \cdot f\left(\cdot, u_{\varepsilon}\right)-\varepsilon \Delta_{\Gamma} u_{\varepsilon}=0 \text { on } G_{T}, \quad u_{\varepsilon}(\cdot, 0)=u_{0 \varepsilon} \text { on } \Gamma_{0} .
$$


If $u_{\varepsilon} \rightarrow u$ a.e. on $G_{T}$ and $u_{0 \varepsilon} \rightarrow u_{0}$ a.e. on $\Gamma_{0}$ for $\varepsilon \rightarrow 0$ and $u \in L^{1}\left(G_{T}\right)$, then $u$ satisfies the entropy condition

$$
-\int_{\Gamma_{0}} \eta\left(u_{0}\right) \phi(\cdot, 0)+\int_{0}^{T} \int_{\Gamma_{t}}\left(-\eta(u) \dot{\phi}(\cdot, t)-q(\cdot, u) \cdot \nabla_{\Gamma} \phi+\nabla_{\Gamma} \cdot v\left(u \eta^{\prime}(u)-\eta(u)\right) \phi\right) \leqslant 0
$$

for all test functions $\phi \in H^{1}\left(G_{T}\right)$ with $\phi \geqslant 0$ and $\phi(\cdot, T)=0$.

Proof. Let $\eta$ and $q$ be defined as above. We multiply (1.1) by $\eta^{\prime}\left(u_{\varepsilon}\right)$ and obtain

$$
\dot{u}_{\varepsilon} \eta^{\prime}\left(u_{\varepsilon}\right)+u_{\varepsilon} \nabla_{\Gamma} \cdot v \eta^{\prime}\left(u_{\varepsilon}\right)+\nabla_{\Gamma} \cdot f\left(\cdot, u_{\varepsilon}\right) \eta^{\prime}\left(u_{\varepsilon}\right)-\varepsilon \Delta_{\Gamma} u_{\varepsilon} \eta^{\prime}\left(u_{\varepsilon}\right)=0 \text { on } G_{T} .
$$

This implies

$$
\eta\left(\dot{u}_{\varepsilon}\right)+u_{\varepsilon} \nabla_{\Gamma} \cdot v \eta^{\prime}\left(u_{\varepsilon}\right)+f_{l}^{\prime}\left(\cdot, u_{\varepsilon}\right) D_{l} u_{\varepsilon} \eta^{\prime}\left(u_{\varepsilon}\right)-\varepsilon \Delta_{\Gamma} \eta\left(u_{\varepsilon}\right)+\varepsilon \eta^{\prime \prime}\left(u_{\varepsilon}\right)\left(D_{l} u_{\varepsilon}\right)^{2}=0 \quad \text { on } G_{T} \text {. }
$$

We multiply by a smooth test function $\phi$ such that $\phi \geqslant 0, \phi(\cdot, T)=0$ and integrate. This gives

$$
\int_{0}^{T} \int_{\Gamma_{t}} \eta\left(\dot{u}_{\varepsilon}\right) \phi+u_{\varepsilon} \nabla_{\Gamma} \cdot v \eta^{\prime}\left(u_{\varepsilon}\right) \phi+f_{l}^{\prime} D_{l} u \eta^{\prime}\left(u_{\varepsilon}\right) \phi-\varepsilon \Delta_{\Gamma} \eta\left(u_{\varepsilon}\right) \phi+\varepsilon \eta^{\prime \prime}\left(u_{\varepsilon}\right)\left(D_{l} u_{\varepsilon}\right)^{2} \phi=0 \text {. }
$$

Since

$$
\begin{aligned}
R & :=-\int_{\Gamma_{0}} \eta\left(u_{\varepsilon}(\cdot, 0)\right) \phi(\cdot, 0)=\int_{0}^{T} \frac{d}{d t} \int_{\Gamma_{t}} \eta\left(u_{\varepsilon}(\cdot, t)\right) \phi(\cdot, t) \\
& =\int_{0}^{T} \int_{\Gamma_{t}}\left(\eta\left(u_{\varepsilon}(\cdot, t)\right) \phi(\cdot, t)\right)^{\cdot}+\int_{0}^{T} \int_{\Gamma_{t}} \eta\left(u_{\varepsilon}\right) \phi \nabla_{\Gamma} \cdot v \\
& =\int_{0}^{T} \int_{\Gamma_{t}}\left(\eta\left(u_{\varepsilon}(\cdot, t)\right)^{\cdot} \phi(\cdot, t)+\eta\left(u_{\varepsilon}(\cdot, t)\right) \dot{\phi}(\cdot, t)\right)+\int_{0}^{T} \int_{\Gamma_{t}} \eta\left(u_{\varepsilon}\right) \phi \nabla_{\Gamma} \cdot v,
\end{aligned}
$$

we obtain from (4.6):

$$
\begin{aligned}
R- & \int_{0}^{T} \int_{\Gamma_{t}} \eta\left(u_{\varepsilon}(\cdot, t)\right) \dot{\phi}(\cdot, t) \\
& -\int_{0}^{T} \int_{\Gamma_{t}}\left(\eta\left(u_{\varepsilon}\right) \phi \nabla_{\Gamma} \cdot v-u_{\varepsilon} \nabla_{\Gamma} \cdot v \eta^{\prime}\left(u_{\varepsilon}\right) \phi-f_{l u} D_{l} u_{\varepsilon} \eta^{\prime}\left(u_{\varepsilon}\right) \phi+\varepsilon \eta\left(u_{\varepsilon}\right) \Delta_{\Gamma} \phi\right) \\
=R- & \int_{0}^{T} \int_{\Gamma_{t}} \eta\left(u_{\varepsilon}(\cdot, t)\right) \dot{\phi}(\cdot, t) \\
& \quad-\int_{0}^{T} \int_{\Gamma_{t}}\left(\eta\left(u_{\varepsilon}\right) \phi \nabla_{\Gamma} \cdot v-u_{\varepsilon} \nabla_{\Gamma} \cdot v \eta^{\prime}\left(u_{\varepsilon}\right) \phi+q\left(u_{\varepsilon}\right) \cdot \nabla_{\Gamma} \phi+\varepsilon \eta\left(u_{\varepsilon}\right) \Delta_{\Gamma} \phi\right) \leqslant 0 .
\end{aligned}
$$

This means that we have the inequality

$$
\begin{aligned}
& -\int_{\Gamma_{0}} \eta\left(u_{0 \varepsilon}\right) \phi(\cdot, 0)-\int_{0}^{T} \int_{\Gamma_{t}} \eta\left(u_{\varepsilon}(\cdot, s)\right) \dot{\phi}(\cdot, s) \\
& \quad-\int_{0}^{T} \int_{\Gamma_{t}}\left(\eta\left(u_{\varepsilon}\right) \phi \nabla_{\Gamma} \cdot v-u_{\varepsilon} \nabla_{\Gamma} \cdot v \eta^{\prime}\left(u_{\varepsilon}\right) \phi+q\left(u_{\varepsilon}\right) \cdot \nabla_{\Gamma} \phi+\varepsilon \eta\left(u_{\varepsilon}\right) \Delta_{\Gamma} \phi\right) \leqslant 0,
\end{aligned}
$$


and for $\varepsilon \rightarrow 0$ we obtain in the limit

$$
\begin{aligned}
& -\int_{\Gamma_{0}} \eta\left(u_{0}\right) \phi(\cdot, 0)-\int_{0}^{T} \int_{\Gamma_{t}} \eta(u(\cdot, s)) \dot{\phi}(\cdot, s) \\
& \quad-\int_{0}^{T} \int_{\Gamma_{t}}\left(\eta(u) \phi \nabla_{\Gamma} \cdot v-u \nabla_{\Gamma} \cdot v \eta^{\prime}(u) \phi+q(u) \cdot \nabla_{\Gamma} \phi\right) \leqslant 0 .
\end{aligned}
$$

This finally proves the Lemma.

Now we use the property (4.4) for the definition of an entropy solution.

Definition 4.3 Let $\eta, q_{l}$ and $u_{0}$ be as in Lemma 4.2. Then $u \in L^{\infty}\left(G_{T}\right)$ is an entropy solution (admissible weak solution) of (1.1) if

$$
-\int_{\Gamma_{0}} \eta\left(u_{0}\right) \phi(\cdot, 0)+\int_{0}^{T} \int_{\Gamma_{t}}\left(-\eta(u) \dot{\phi}(\cdot, t)-q(\cdot, u) \cdot \nabla_{\Gamma} \phi+\phi \nabla_{\Gamma} \cdot v\left(u \eta^{\prime}(u)-\eta(u)\right)\right) \leqslant 0
$$

holds for all test functions $\phi \in H^{1}\left(G_{T}\right)$ with $\phi \geqslant 0$ and $\phi(\cdot, T)=0$ and for all $\eta$ and $q$ with the properties, mentioned above.

REMARK 4.4 If we choose $\eta(u)=u$ in Definition 4.3, then this implies $q(\cdot, u)=f(\cdot, u)+$ const, $u \eta^{\prime}(u)-\eta(u)=0$ and that $u$ is a weak solution of (1.1).

The following definition of Kruzkov entropy solutions is equivalent to Definition 4.3.

DEFINITION 4.5 A function $u \in L^{\infty}\left(G_{T}\right)$ is called Kruzkov entropy solution of (1.1) if

$$
\begin{aligned}
\int_{0}^{T} \int_{\Gamma}|u-k| \dot{\varphi}-\operatorname{sign}(u-k) k \nabla_{\Gamma} \cdot v \varphi+\operatorname{sign}(u-k)(f(\cdot, u)-f(\cdot, k)) \cdot \nabla_{\Gamma} \varphi & \\
& +\int_{\Gamma_{0}}\left|u_{0}-k\right| \varphi(\cdot, 0) \geqslant 0
\end{aligned}
$$

for all $k \in \mathbb{R}$ and all test functions $\varphi \in C^{1}\left(\overline{G_{T}}\right)$ with $\varphi \geqslant 0$ and $\varphi(\cdot, T)=0$.

REMARK 4.6 An entropy solution is a Kruzkov entropy solution. This can be seen by a regularization of the Kruzkov entropy-entropy flux pair. See for example [24].

\section{The regularized problem}

In order to solve the conservation law (1.1) we solve the initial value problem (4.3) and consider $u_{\varepsilon}$ for $\varepsilon \rightarrow 0$. For technical reasons let us consider the following regularized PDE

$$
\dot{u}_{\varepsilon}+u_{\varepsilon} \nabla_{\Gamma} \cdot v+\nabla_{\Gamma} \cdot f\left(\cdot, u_{\varepsilon}\right)-\varepsilon \nabla_{\Gamma} \cdot\left(B \nabla_{\Gamma} u_{\varepsilon}\right)=0
$$

on $G_{T}$ with initial data $u_{\varepsilon}(\cdot, 0)=u_{0 \varepsilon}$ on $\Gamma_{0}$ with $u_{0 \varepsilon} \rightarrow u_{0}$ a.e. on $\Gamma_{0}$ and (4.2). Here $B=B(x, t)$ is a symmetric diffusion matrix which maps the tangent space of $\Gamma(t)$ into the tangent space at the point $x \in \Gamma(t)$, so that we have $B v=0$ and $v^{*} B=0$. Assume also that $B$ is positive definite on the tangent space. Similarly as in Lemma 4.2 it can be shown that $u$ is an entropy solution if $u_{\varepsilon} \rightarrow u$ for $\varepsilon \rightarrow 0$. In the proofs of the following Section we will use the fact that

$$
B P=P B=B .
$$

The main purpose of the next section is to prove a priori bounds for $u_{\varepsilon}$ which are independent of $\varepsilon$. 


\section{A priori estimates for the regularized problem}

In this section we replace $u_{\varepsilon}$ by $u$ for better readability. The aim of this section is the derivation of a priori estimates which are independent of $\varepsilon$. The initial value problem

$$
\dot{u}+u \nabla_{\Gamma} \cdot v+\nabla_{\Gamma} \cdot f(\cdot, u)-\varepsilon \nabla_{\Gamma} \cdot\left(B \nabla_{\Gamma} u\right)=0, \quad u(\cdot, 0)=u_{0 \varepsilon}
$$

has a unique smooth solution. This is shown by dovetailing the cut-off technique of Kruzkov with the Galerkin ansatz from [14]. The proof is quite straight forward and so we omit the details here. The proof of smoothness of the weak solution found by this method is a purely local argument.

\subsection{Estimate of the solution}

We prove that the solution $u$ of the regularized parabolic initial value problem (6.1) is bounded in the $L^{\infty}$-norm in space and time independently of the parameter $\varepsilon$.

LEMMA 6.1 Let $u$ be the solution of (6.1). Then

$$
\sup _{t \in(0, T)}\|u(\cdot, t)\|_{L^{\infty}(\Gamma(t))} \leqslant c
$$

with a constant $c$ which is independent of $\varepsilon$ but depends on the data of the problem including the final time $T$ and $c_{0}$ from (4.2).

Proof. This estimate is a consequence of the maximum principle for parabolic PDEs. Because of the unusual setting here, we give a proof. The PDE (5.1) can be written in a weak form. Note that the nonlinearity $f((x, t), u)$ is tangentially divergence free with respect to the $x$-variable. This is crucial here. We begin by transforming $u$ :

$$
w(x, t)=e^{-\lambda t} u(x, t), \quad x \in \Gamma(t),
$$

where we set $\lambda=\sup _{(0, T)}\left\|\left(\nabla_{\Gamma} \cdot v\right)_{-}\right\|_{L^{\infty}(\Gamma)}$ with $\left(\nabla_{\Gamma} \cdot v\right)_{-}=\min \left\{\nabla_{\Gamma} \cdot v, 0\right\}$. We set $g(\cdot, w)=$ $e^{-\lambda t} f\left(\cdot, e^{\lambda t} w\right) \psi(w)$ with a function $\psi \in C_{0}^{1}(\mathbb{R})$ which satisfies $\psi(w)=1$ for $|w| \leqslant M_{\varepsilon}$ and $\psi(w)=0$ for $|w| \geqslant M_{\varepsilon}+1$. We use $M_{\varepsilon}=\|u\|_{L^{\infty}\left(G_{T}\right)}$. Then

$$
\left|\frac{\partial g}{\partial w}(\cdot, w)\right| \leqslant c\left(M_{\varepsilon}\right) \text {. }
$$

Because of $|w|=e^{-\lambda t}|u| \leqslant M_{\varepsilon}$ we then have

$$
\int_{\Gamma} \dot{w} \varphi+\int_{\Gamma} w \varphi\left(\lambda+\nabla_{\Gamma} \cdot v\right)-\int_{\Gamma} g(\cdot, w) \cdot \nabla_{\Gamma} \varphi+\varepsilon \int_{\Gamma} B \nabla_{\Gamma} w \cdot \nabla_{\Gamma} \varphi=0
$$

for every $\varphi$. If we choose $\varphi=(w-M)_{+}=\max \{w-M, 0\}$ with $M=c_{0} \geqslant\left\|u_{0 \varepsilon}\right\|_{L^{\infty}\left(\Gamma_{0}\right)}$ in this equation, then we arrive at

$$
\begin{aligned}
\frac{1}{2} \int_{\Gamma}\left((w-M)_{+}^{2}\right) & +\varepsilon \int_{\Gamma} B \nabla_{\Gamma}(w-M)_{+} \cdot \nabla_{\Gamma}(w-M)_{+} \\
= & \int_{\Gamma}(g(\cdot, w)-g(\cdot, M)) \cdot \nabla_{\Gamma}(w-M)_{+}-\int_{\Gamma} w(w-M)_{+}\left(\lambda+\nabla_{\Gamma} \cdot v\right) .
\end{aligned}
$$


Here we have used that $g(\cdot, M) \cdot v=0$. For the right hand side of this equation we observe that for our choice of $\lambda$

$$
w(w-M)_{+}\left(\lambda+\nabla_{\Gamma} \cdot v\right) \geqslant 0,
$$

and that

$$
|g(\cdot, w)-g(\cdot, M)| \leqslant c\left(M_{\varepsilon}\right)|w-M| .
$$

We use these two estimates together with the ellipticity condition and get with a positive constant $c$ the estimate

$$
\begin{aligned}
& \frac{1}{2} \frac{d}{d t} \int_{\Gamma}(w-M)_{+}^{2}+\varepsilon c \int_{\Gamma}\left|\nabla_{\Gamma}(w-M)_{+}\right|^{2} \\
& \quad \leqslant \frac{1}{2}\left\|\left(\nabla_{\Gamma} \cdot v\right)_{+}\right\|_{L^{\infty}(\Gamma)} \int_{\Gamma}(w-M)_{+}^{2}+c\left(M_{\varepsilon}\right) \int_{\Gamma}(w-M)_{+}\left|\nabla_{\Gamma}(w-M)_{+}\right| .
\end{aligned}
$$

Here we also have used the transport theorem from Lemma 3.1. From the previous estimate we infer with Young's inequality that

$$
\frac{d}{d t} \int_{\Gamma}(w-M)_{+}^{2}+\varepsilon c \int_{\Gamma}\left|\nabla_{\Gamma}(w-M)_{+}\right|^{2} \leqslant c\left(M_{\varepsilon}, \varepsilon\right) \int_{\Gamma}(w-M)_{+}^{2} .
$$

This implies for the nonnegative function $\phi(t)=\int_{\Gamma(t)}(w(\cdot, t)-M)_{+}^{2}$ the inequality

$$
\phi^{\prime}(t) \leqslant c\left(M_{\varepsilon}, \varepsilon\right) \phi(t) .
$$

Because of $\phi(0)=0$ we then obtain with a Gronwall argument that $\phi(t)=0$. But this says that $(w-M)_{+}=0$ almost everywhere which implies $w \leqslant M$ or

$$
u(\cdot, t) \leqslant c(T, M) \text { on } \Gamma(t)
$$

and the constant $c(T, M)$ does not depend on $\varepsilon$. The estimate from below follows similarly.

\subsection{Estimate of the spatial gradient}

LEMma 6.2 Assume that $u$ solves the regularized PDE (6.1). Then

$$
\sup _{(0, T)} \int_{\Gamma}\left|\nabla_{\Gamma} u\right| \leqslant c
$$

with a constant $c$ which does not depend on $\varepsilon$.

Proof. Set $w_{i}=\underline{D}_{i} u$ and $w=\left(w_{1}, \ldots, w_{n+1}\right)$. We take the derivative $\underline{D}_{i}$ of the regularized PDE (6.1),

$$
\underline{D}_{i} \dot{u}+\underline{D}_{i}\left(u \nabla_{\Gamma} \cdot v\right)+\underline{D}_{i} \nabla_{\Gamma} \cdot(f(\cdot, u))-\varepsilon \underline{D}_{i} \nabla_{\Gamma} \cdot\left(B \nabla_{\Gamma} u\right)=0
$$

and treat the terms separately. With the equation (2.6) we get

$$
\underline{D}_{i} \dot{u}=\left(\underline{D}_{i} u\right)^{\cdot}+A_{i r}(v) \underline{D}_{r} u=\dot{w}_{i}+A_{i r}(v) w_{r} .
$$

Clearly

$$
\underline{D}_{i}\left(u \nabla_{\Gamma} \cdot v\right)=w_{i} \nabla_{\Gamma} \cdot v+u \underline{D}_{i} \nabla_{\Gamma} \cdot v .
$$


For the nonlinear term we get with the use of (2.4)

$$
\begin{aligned}
\underline{D}_{i} \nabla_{\Gamma} \cdot(f(\cdot, u))= & \underline{D}_{i}\left(\underline{D}_{k} f_{k}(\cdot, u)+f_{k u}(\cdot, u) \underline{D}_{k} u\right) \\
= & \underline{D}_{i} \underline{D}_{k} f_{k}(\cdot, u)+\underline{D}_{k} f_{k u}(\cdot, u) w_{i}+\underline{D}_{i} f_{k u}(\cdot, u) w_{k} \\
& \quad+f_{k u u}(\cdot, u) w_{i} w_{k}+f_{k u}(\cdot, u)\left(\underline{D}_{k} w_{i}+\mathcal{H}_{k l} w_{l} v_{i}-\mathcal{H}_{i l} w_{l} v_{k}\right) .
\end{aligned}
$$

With (2.4) the second order term can be rewritten as follows:

$$
\begin{aligned}
& \underline{D}_{i} \nabla_{\Gamma} \cdot\left(B \nabla_{\Gamma} u\right)= \underline{D}_{i} \underline{D}_{k}\left(B_{k m} \underline{D}_{m} u\right) \\
&= \underline{D}_{k} \\
&=\underline{D}_{i}\left(B_{k m} \underline{D}_{m} u\right)+\mathcal{H}_{k l} \underline{D}_{l}\left(B_{k m} \underline{D}_{m} u\right) v_{i}-\mathcal{H}_{i l} \underline{D}_{l}\left(B_{k m} \underline{D}_{m} u\right) v_{k} \\
& \quad \underline{D}_{k}\left(\underline{D}_{i} B_{k m} \underline{D}_{m} u\right)+\underline{D}_{k}\left(B_{k m}\left(\underline{D}_{m} \underline{D}_{i} u+\mathcal{H}_{m l} \underline{D}_{l} u v_{i}-\mathcal{H}_{i l} \underline{D}_{l} u v_{m}\right)\right) \\
& \quad \quad+\mathcal{H}_{k l} \underline{D}_{l}\left(B_{k m} \underline{D}_{m} u\right) v_{i}-\mathcal{H}_{i l} \underline{D}_{l}\left(B_{k m} \underline{D}_{m} u\right) v_{k} \\
&=\underline{D}_{k}\left(\underline{D}_{i} B_{k m} w_{m}\right)+\underline{D}_{k}\left(B_{k m}\left(\underline{D}_{m} w_{i}+\mathcal{H}_{m l} w_{l} v_{i}-\mathcal{H}_{i l} w_{l} v_{m}\right)\right) \\
& \quad+\mathcal{H}_{k l} \underline{D}_{l}\left(B_{k m} w_{m}\right) v_{i}-\mathcal{H}_{i l} \underline{D}_{l}\left(B_{k m} w_{m}\right) v_{k} \\
&=\underline{D}_{k}\left(B_{k m} \underline{D}_{m} w_{i}\right)+\underline{D}_{k}\left(\underline{D}_{i} B_{k m} w_{m}\right)+\underline{D}_{k}\left(B_{k m}\left(\mathcal{H}_{m l} w_{l} v_{i}-\mathcal{H}_{i l} w_{l} v_{m}\right)\right) \\
& \quad+\mathcal{H}_{k l} \underline{D}_{l}\left(B_{k m} w_{m}\right) v_{i}-\mathcal{H}_{i l} \underline{D}_{l}\left(B_{k m} w_{m}\right) v_{k} \\
&=\underline{D}_{k}\left(B_{k m} \underline{D}_{m} w_{i}\right)+\underline{D}_{k}\left(\underline{D}_{i} B_{k m} w_{m}\right)+\underline{D}_{k}\left(B_{k m} \mathcal{H}_{m l} w_{l}\right) v_{i} \\
& \quad+B_{k m} \mathcal{H}_{m l} \mathcal{H}_{i k} w_{l}+\mathcal{H}_{k l} \underline{D}_{l}\left(B_{k m} w_{m}\right) v_{i}+\mathcal{H}_{i l} \mathcal{H}_{l k} B_{k m} w_{m} .
\end{aligned}
$$

For the last term we have used $\mathcal{H}_{i l} \underline{D}_{l}\left(B_{k m} w_{m}\right) v_{k}=-\mathcal{H}_{i l} \mathcal{H}_{l k} B_{k m} w_{m}$. We now collect the intermediate results (6.6), (6.7), (6.8) and (6.9) to arrive at the following PDE for $w_{i}=\underline{D}_{i} u$.

$$
\begin{gathered}
\dot{w}_{i}+A_{i r}(v) w_{r}+w_{i} \nabla_{\Gamma} \cdot v+u \underline{D}_{i} \nabla_{\Gamma} \cdot v+\underline{D}_{i} \underline{D}_{k} f_{k}(\cdot, u)+\underline{D}_{k} f_{k u}(\cdot, u) w_{i} \\
+\underline{D}_{i} f_{k u}(\cdot, u) w_{k}+f_{k u u}(\cdot, u) w_{i} w_{k}+f_{k u}(\cdot, u)\left(\underline{D}_{k} w_{i}+\mathcal{H}_{k l} w_{l} v_{i}-\mathcal{H}_{i l} w_{l} v_{k}\right) \\
-\varepsilon\left(\underline{D}_{k}\left(B_{k m} \underline{D}_{m} w_{i}\right)+\underline{D}_{k}\left(\underline{D}_{i} B_{k m} w_{m}\right)+\underline{D}_{k}\left(B_{k m} \mathcal{H}_{m l} w_{l}\right) v_{i}\right) \\
\quad-\varepsilon\left(\mathcal{H}_{i k} \mathcal{H}_{m l} B_{k m} w_{l}+\mathcal{H}_{k l} \underline{D}_{l}\left(B_{k m} w_{m}\right) v_{i}+\mathcal{H}_{i l} \mathcal{H}_{l k} B_{k m} w_{m}\right)=0 .
\end{gathered}
$$

We multiply this equation by $\frac{w_{i}}{|w|}$, sum with respect to $i$ from 1 to $n+1$, use the fact that $w$ is a tangent vector, and get

$$
\begin{aligned}
|w| \cdot+|w| \nabla_{\Gamma} \cdot v+ & |w| \underline{D}_{k} f_{k u}(\cdot, u)+f_{k u u}(\cdot, u)|w| w_{k}+f_{k u}(\cdot, u) \frac{w_{i}}{|w|} \underline{D}_{k} w_{i} \\
& +\frac{w_{i}}{|w|} \underline{D}_{i} \underline{D}_{k} f_{k}(\cdot, u)+\underline{D}_{i} f_{k u}(\cdot, u) \frac{w_{i}}{|w|} w_{k}-\varepsilon \frac{w_{i}}{|w|} \underline{D}_{k}\left(B_{k m} \underline{D}_{m} w_{i}\right) \\
=-\underline{D}_{i} v_{r} \frac{w_{i}}{|w|} w_{r}+ & \varepsilon \frac{w_{i}}{|w|} \underline{D}_{k}\left(\underline{D}_{i} B_{k m} w_{m}\right) \\
& +\varepsilon B_{k m} \mathcal{H}_{m l} \mathcal{H}_{i k} w_{l} \frac{w_{i}}{|w|}+\varepsilon B_{k m} \mathcal{H}_{i l} \mathcal{H}_{l k} w_{m} \frac{w_{i}}{|w|}-u \frac{w_{i}}{|w|} \underline{D}_{i} \nabla_{\Gamma} \cdot v .
\end{aligned}
$$

Here we also used that $f_{k u}(\cdot, u) v_{k}=0$ and $w_{i} v_{i}=0$. We now observe that

$$
\underline{D}_{k} f_{k u}(\cdot, u)|w|+f_{k u u}(\cdot, u) w_{k}|w|+f_{k u}(\cdot, u) \frac{w_{i}}{|w|} \underline{D}_{k} w_{i}=\underline{D}_{k}\left(f_{k u}(\cdot, u)|w|\right) .
$$


The result of Lemma 6.1 allows us to estimate some terms in (6.10).

$$
\begin{aligned}
|w| \cdot+|w| \nabla_{\Gamma} \cdot v+\nabla_{\Gamma} \cdot\left(f_{u}(\cdot, u)|w|\right) & -\varepsilon \frac{w_{i}}{|w|} \underline{D}_{k}\left(B_{k m} \underline{D}_{m} w_{i}\right) \\
& \leqslant c_{1}+c_{2}|w|+c_{3}|u|+\varepsilon \frac{w_{i}}{|w|} \underline{D}_{k}\left(\underline{D}_{i} B_{k m} w_{m}\right) .
\end{aligned}
$$

We rewrite the second order term on the left hand side of this equation (integrated over $\Gamma$ ).

$$
\begin{aligned}
-\varepsilon \int_{\Gamma} \frac{w_{i}}{|w|} \underline{D}_{k}\left(B_{k m} \underline{D}_{m} w_{i}\right) & =-\varepsilon \int_{\Gamma} \frac{w_{i}}{|w|} H v_{k} B_{k m} \underline{D}_{m} w_{i}+\varepsilon \int_{\Gamma} \underline{D}_{k}\left(\frac{w_{i}}{|w|}\right) B_{k m} \underline{D}_{m} w_{i} \\
& =\varepsilon \int_{\Gamma} \frac{1}{|w|} Q_{i l} B_{k m} \underline{D}_{k} w_{l} \underline{D}_{m} w_{i}
\end{aligned}
$$

where we have set

$$
Q_{i l}=\delta_{i l}-\frac{w_{i} w_{l}}{|w|^{2}}, \quad i, l=1, \ldots, n+1
$$

We now estimate the last term on the right hand side of (6.12) integrated over $\Gamma$.

$$
\begin{aligned}
\varepsilon \int_{\Gamma} \frac{w_{i}}{|w|} \underline{D}_{k}\left(\underline{D}_{i} B_{k m} w_{m}\right) & =\varepsilon \int_{\Gamma} \frac{w_{i}}{|w|} H v_{k} \underline{D}_{i} B_{k m} w_{m}-\varepsilon \int_{\Gamma} \frac{1}{|w|} Q_{i l} \underline{D}_{k} w_{l} \underline{D}_{i} B_{k m} w_{m} \\
& =-\varepsilon \int_{\Gamma} \frac{w_{i}}{|w|} H B_{k m} \mathcal{H}_{i k} w_{m}-\varepsilon \int_{\Gamma} \frac{1}{|w|} Q_{i l} \underline{D}_{i} B_{k m} w_{m} \underline{D}_{k} w_{l} \\
& \leqslant c_{4} \varepsilon \int_{\Gamma}|w|-\varepsilon \int_{\Gamma} \frac{1}{|w|} Q_{i l} \underline{D}_{i} B_{k m} w_{m} \underline{D}_{k} w_{l} .
\end{aligned}
$$

Since the matrix $Q$ is positive semidefinite and since $B$ is positive definite on the tangent space we can estimate the last term on the right hand side. We use the abbreviations

$$
z_{r l}=\underline{D}_{r} w_{l}, \quad \zeta_{i s}=B_{s k}^{-1} P_{k p} \underline{D}_{i} B_{p m} w_{m} .
$$

For any $\delta>0$ we then have

$$
\begin{aligned}
\left|Q_{i l} \underline{D}_{i} B_{k m} \underline{D}_{k} w_{l} w_{m}\right|=\left|Q_{i l} B_{r s} z_{r l} \zeta_{i s}\right| & \leqslant \sqrt{Q_{i l} B_{r s} z_{r l} z_{i s}} \sqrt{Q_{i l} B_{r s} \zeta_{i s} \zeta_{r l}} \\
& \leqslant \frac{\delta}{2} Q_{i l} B_{r s} z_{r l} z_{i s}+\frac{1}{2 \delta} Q_{i l} B_{r s} \zeta_{i s} \zeta_{r l} \\
& \leqslant \frac{\delta}{2} Q_{i l} B_{r s} \underline{D}_{r} w_{l} \underline{D}_{i} w_{s}+c(\delta)|w|^{2} .
\end{aligned}
$$

The first equation can be obtained as follows:

$$
\begin{aligned}
Q_{i l} B_{r s} z_{r l} \zeta_{i s} & =Q_{i l} B_{r s} \underline{D}_{r} w_{l} B_{s k}^{-1} P_{k p} \underline{D}_{i} B_{p m} w_{m}=Q_{i l} \underline{D}_{r} w_{l} P_{k p} \underline{D}_{i} B_{p m} w_{m} \delta_{r k} \\
& =Q_{i l} \underline{D}_{r} w_{l} P_{r p} \underline{D}_{i} B_{p m} w_{m}=Q_{i l} \underline{D}_{r} w_{l}\left(\delta_{r p}-v_{r} v_{p}\right) \underline{D}_{i} B_{p m} w_{m} \\
& =Q_{i l} \underline{D}_{p} w_{l} \underline{D}_{i} B_{p m} w_{m} .
\end{aligned}
$$

Now because of

$$
\underline{D}_{i} w_{s}=\underline{D}_{s} w_{i}+\mathcal{H}_{s p} w_{p} v_{i}-\mathcal{H}_{i p} w_{p} v_{s}
$$


we get

$$
Q_{i l} B_{r s} \underline{D}_{r} w_{l} \underline{D}_{i} w_{s}=Q_{i l} B_{r s} \underline{D}_{r} w_{l} \underline{D}_{s} w_{i}+Q_{i l} B_{r s} \mathcal{H}_{s p} v_{i} \underline{D}_{r} w_{l} w_{p}
$$

and because of $Q_{i l} v_{i}=v_{l}$, the second term on the right hand side can be rewritten as follows:

$$
\begin{aligned}
Q_{i l} B_{r s} \mathcal{H}_{s p} v_{i} \underline{D}_{r} w_{l} w_{p} & =v_{l} B_{r s} \mathcal{H}_{s p} v_{i} \underline{D}_{r} w_{l} w_{p} \\
& =B_{r s} \mathcal{H}_{s p} v_{i}\left(\underline{D}_{r}\left(w_{l} v_{l}\right)-w_{l} \mathcal{H}_{r l}\right) w_{p} \\
& =-B_{r s} \mathcal{H}_{s p} \mathcal{H}_{r l} v_{i} w_{l} w_{p} .
\end{aligned}
$$

Collecting the previous estimates we arrive at

$$
\varepsilon \int_{\Gamma} \frac{w_{i}}{|w|} \underline{D}_{k}\left(\underline{D}_{i} B_{k m} w_{m}\right) \leqslant c \varepsilon \int_{\Gamma}|w|+\frac{\varepsilon}{2} \int_{\Gamma} \frac{1}{|w|} Q_{i l} B_{k m} \underline{D}_{k} w_{l} \underline{D}_{m} w_{i}
$$

We integrate (6.12) over $\Gamma$ and finally get

$$
\begin{aligned}
\int_{\Gamma}|w|^{\cdot}+|w| \nabla_{\Gamma} \cdot v+\int_{\Gamma} \nabla_{\Gamma} \cdot\left(f_{u}(\cdot, u)|w|\right) & \leqslant-\frac{\varepsilon}{2} \int_{\Gamma} \frac{1}{|w|} Q_{i l} B_{k m} \underline{D}_{k} w_{l} \underline{D}_{m} w_{i}+c \int_{\Gamma}|w| \\
& \leqslant c \int_{\Gamma}|w|+c \int_{\Gamma}|u|+c \int_{\Gamma}|u|+c,
\end{aligned}
$$

where we again have used the fact that $\Gamma$ is compact. Since

$$
\int_{\Gamma} \nabla_{\Gamma} \cdot(f(\cdot, u)|w|)=\int_{\Gamma} H v \cdot f(\cdot, u)|w|=0,
$$

we finally get the estimate

$$
\frac{d}{d t} \int_{\Gamma}|w| \leqslant c \int_{\Gamma}|w|+c .
$$

Here we have used the bound (6.2) for $u$. In summary we have proved that

$$
\sup _{(0, T)} \int_{\Gamma}|w| \leqslant c
$$

independently of $\varepsilon$. In the last step we used (4.2). Lemma 6.2 is proved.

\section{Estimate of the time derivative}

Assume that the matrix $B=\left(B_{i k}\right)_{i, k=1, \ldots, n+1}$ satisfies

$$
\dot{B}=B A(v)+A(v)^{*} B+\lambda B, B(\cdot, 0)=B_{0}
$$

where $\lambda>0$ is a constant and $B_{0}$ is a symmetric and tangentially positive definite $(n+1) \times(n+1)$ matrix.

LEMMA 7.1 There is a symmetric $(n+1) \times(n+1)$ matrix which is positive definite on the tangent space and solves (7.1). 
Proof. The existence of $B$ follows easily from ODE theory, since (7.1) is a linear transport equation.

Let us show, that $B$ is symmetric. The transposed matrix solves the ODE

$$
\dot{B}^{*}=A(v)^{*} B^{*}+B^{*} A(v)+\lambda B^{*} .
$$

If we subtract this equation from (7.1), we get

$$
\left(B-B^{*}\right)^{\cdot}=\left(B-B^{*}\right) A(v)+A(v)^{*}\left(B-B^{*}\right)+\lambda\left(B-B^{*}\right) .
$$

Since $\left(B-B^{*}\right)(\cdot, 0)=0$ by assumption, we have that $B-B^{*}=0$ for all times.

The coercivity is seen as follows.

$$
\left(e^{-\lambda t} B\right)^{\cdot}=e^{-\lambda t}(\dot{B}-\lambda B)=e^{-\lambda t}\left(B A(v)+A(v)^{*} B\right) .
$$

Thus

$$
B(\cdot, t)=e^{\lambda t}\left(B_{0}+\int_{0}^{t} e^{-\lambda s}\left(B A(v)+A(v)^{*} B\right) d s\right)
$$

and from this we get for $\xi \in \mathbb{R}^{n+1}$ with the coercivity of $B_{0}, B_{0} \xi \cdot \xi \geqslant d_{0}|\xi|^{2}\left(d_{0}>0\right)$, and the smoothness of $A(v)$ and $B$ :

$$
B(\cdot, t) \xi \cdot \xi \geqslant e^{\lambda t} B_{0} \xi \cdot \xi-c e^{\lambda t}|\xi|^{2} \int_{0}^{t} e^{-\lambda s} d s \geqslant\left(e^{\lambda t}\left(d_{0}-\frac{c}{\lambda}\right)+\frac{c}{\lambda}\right)|\xi|^{2} .
$$

So, $\mathrm{B}$ is positive definite if we choose $\lambda$ big enough. Now observe that $\tilde{B}=P B P$ is also a solution of the ODE by using $\dot{P}=[P, A(v)]$ which can be seen by $\dot{v}_{k}=-v_{l} \underline{D}_{k} v_{l}$ and where $[\cdot, \cdot]$ is the commutator. We denote $\tilde{B}$ again by $B$.

LEMma 7.2 Assume that $u$ solves the regularized PDE (5.1). Then

$$
\sup _{(0, T)} \int_{\Gamma}|\dot{u}| \leqslant c
$$

with a constant $c$ which does not depend on $\varepsilon$.

Proof. We take the material derivative of (6.1) and set $z=\dot{u}$,

$$
\ddot{u}+\dot{u} \nabla_{\Gamma} \cdot v+u\left(\nabla_{\Gamma} \cdot v\right) \dot{+}\left(\nabla_{\Gamma} \cdot(f(\cdot, u))\right) \cdot-\varepsilon\left(\nabla_{\Gamma} \cdot\left(B \nabla_{\Gamma} u\right)\right)^{\cdot}=0 .
$$

We use (2.6) and treat the terms separately. Clearly

$$
\ddot{u}+\dot{u} \nabla_{\Gamma} \cdot v=\dot{z}+z \nabla_{\Gamma} \cdot v .
$$

For the nonlinear terms we get

$$
\begin{aligned}
\left(\nabla_{\Gamma} \cdot f(\cdot, u)\right)^{\cdot}= & \left(\underline{D}_{l} f_{l}(\cdot, u)+f_{l u}(\cdot, u) \underline{D}_{l} u\right)^{\cdot} \\
= & \underline{D}_{l} \cdot f_{l}(\cdot, u)-A_{l r}(v) \underline{D}_{r} f_{l}(\cdot, u)+\dot{f}_{l u}(\cdot, u) \underline{D}_{l} u+f_{l u u}(\cdot, u) z \underline{D}_{l} u \\
& +f_{l u}(\cdot, u)\left(\underline{D}_{l} z-A_{l r}(v) \underline{D}_{r} u\right) .
\end{aligned}
$$


We calculate the material derivative of the second order term.

$$
\begin{aligned}
\left(\nabla_{\Gamma} \cdot\left(B \nabla_{\Gamma} u\right)\right)^{\cdot}= & \left(\underline{D}_{k}\left(B_{k l} \underline{D}_{l} u\right)\right)^{\cdot} \\
= & \underline{D}_{k}\left(B_{k l} \underline{D}_{l} u\right)^{\cdot}-A_{k r}(v) \underline{D}_{r}\left(B_{k l} \underline{D}_{l} u\right) \\
= & \underline{D}_{k}\left(B_{k l}\left(\underline{D}_{l} z-A_{l r}(v) \underline{D}_{r} u\right)+\dot{B}_{k l} \underline{D}_{l} u\right)-A_{k r}(v) \underline{D}_{r}\left(B_{k l} \underline{D}_{l} u\right) \\
= & \nabla_{\Gamma} \cdot\left(B \nabla_{\Gamma} z\right)-\underline{D}_{k}\left(\left(B_{k r} A_{r l}(v)-\dot{B}_{k l}+A_{r k}(v) B_{r l}\right) \underline{D}_{l} u\right) \\
& \quad+B_{r l} \underline{D}_{l} u \underline{D}_{k}\left(A_{r k}(v)\right)
\end{aligned}
$$

Now choose the matrix $B$ such that it satisfies (7.1). Then

$$
\left(\nabla_{\Gamma} \cdot\left(B \nabla_{\Gamma} u\right)\right)^{\cdot}=\nabla_{\Gamma} \cdot\left(B \nabla_{\Gamma} z\right)+\lambda \underline{D}_{k}\left(B_{k l} \underline{D}_{l} u\right)+B_{r l} \underline{D}_{l} u \underline{D}_{k}\left(A_{r k}(v)\right) .
$$

We collect the terms (7.3), (7.4) and (7.5) to get

$$
\begin{gathered}
\dot{z}+z \nabla_{\Gamma} \cdot v+u\left(\nabla_{\Gamma} \cdot v\right)^{\cdot}+\underline{D}_{l} \dot{f}_{l}(\cdot, u)-A_{l r}(v) \underline{D}_{r} f_{l}(\cdot, u)+\dot{f}_{l u}(\cdot, u) \underline{D}_{l} u \\
+f_{l u u}(\cdot, u) z \underline{D}_{l} u+f_{l u}(\cdot, u) \underline{D}_{l} z-A_{l r}(v) f_{l u}(\cdot, u) \underline{D}_{r} u \\
-\varepsilon \nabla_{\Gamma} \cdot\left(B \nabla_{\Gamma} z\right)-\varepsilon B_{r l} \underline{D}_{l} u \underline{D}_{k}\left(A_{k r}(v)\right)-\lambda\left(z+u \nabla_{\Gamma} \cdot v+\nabla_{\Gamma} \cdot f(\cdot, u)\right)=0 .
\end{gathered}
$$

We observe that similarly as in (6.11) we have

$$
\frac{z}{|z|}\left(f_{l u}(\cdot, u) \underline{D}_{l} z+z f_{l u u}(\cdot, u) \underline{D}_{l} u\right)=\nabla_{\Gamma} \cdot\left(f_{u}(\cdot, u)|z|\right)-\underline{D}_{l} f_{l u}(\cdot, u)|z| .
$$

Multiplying (7.6) with $\operatorname{sign}(z)$ we get (correctly: use $\frac{z}{\sqrt{\delta^{2}+z^{2}}}$ for $\delta \rightarrow 0$ etc.)

$$
|z|^{\cdot}+|z| \nabla_{\Gamma} \cdot v+\nabla_{\Gamma} \cdot\left(f_{u}(\cdot, u)|z|\right) \leqslant \varepsilon \operatorname{sign}(z) \nabla_{\Gamma} \cdot\left(B \nabla_{\Gamma} z\right)+c_{1}+c_{2}\left|\nabla_{\Gamma} u\right|+c_{3}|z| .
$$

Here we have used the boundedness of $u$ uniformly with respect to the regularization parameter $0<\varepsilon \leqslant 1$. With the same arguments as in the proof of Lemma 6.2 after integration over $\Gamma$ we get the inequality

$$
\frac{d}{d t} \int_{\Gamma}|z| \leqslant c_{1}+c_{2} \int_{\Gamma}\left|\nabla_{\Gamma} u\right|+c_{3} \int_{\Gamma}|z|,
$$

and Lemma 6.2 implies

$$
\sup _{(0, T)} \int_{\Gamma}|z| \leqslant c
$$

with a constant $c$, which does not depend on $\varepsilon$, since we can use (4.2).

\section{Existence for the conservation law}

TheOrem 8.1 Assume Assumptions 2.1, (7.1) and let $u_{0} \in L^{\infty}\left(\Gamma_{0}\right)$. Then there exists an entropy solution of (1.1).

Proof. Let $u_{\varepsilon}$ be the solution of (5.1) with $u_{\varepsilon}(\cdot, 0)=u_{0 \varepsilon}$ on $\Gamma_{0}$ and $u_{0 \varepsilon} \rightarrow u_{0}$ a.e. on $\Gamma_{0}, \Phi$ as in Assumption 2.1, and

$$
w_{\varepsilon}(y, t):=u_{\varepsilon}(\Phi(y, t), t)
$$


for $y \in \Gamma_{0}$ and $t \in[0, T]$. Then due to the Lemmata 6.2 and 7.2 and the properties of $\Phi$ (see Assumption 2.1) we obtain

$$
\begin{aligned}
& \int_{0}^{T} \int_{\Gamma_{0}}\left|\frac{\partial}{\partial t} w_{\varepsilon}\right| \leqslant \text { const. } \\
& \int_{0}^{T} \int_{\Gamma_{0}}\left|\nabla_{\Gamma_{0}} w_{\varepsilon}\right| \leqslant \text { const. }
\end{aligned}
$$

uniformly in $\varepsilon$. This implies that $w_{\varepsilon}$ is uniformly bounded in $H^{1,1}\left(\Gamma_{0} \times[0, T]\right)$. Then due to the Kondrakov-Theorem (see Aubin [3]) we have a convergent subsequence $w_{\varepsilon^{\prime}}$ and $w \in L^{1}\left(\Gamma_{0} \times\right.$ $[0, T])$ such that

$$
w_{\mathcal{E}^{\prime}} \rightarrow w \text { in } L^{1}\left(\Gamma_{0} \times[0, T]\right) .
$$

Define $\Phi_{t}(x):=\Phi(x, t)$. Since $u_{\varepsilon}(x, t)=w_{\varepsilon}\left(\Phi_{t}^{-1}(x), t\right)$ and due to the properties of $\Phi$ (see Assumption 2.1) we have

$$
u_{\varepsilon^{\prime}}(x, t) \rightarrow u(x, t):=w\left(\Phi_{t}^{-1}(x), t\right) \quad \text { in } \quad L^{1}\left(G_{T}\right) .
$$

Then we proceed as in the proof for Lemma 4.2 to prove that $u$ is an entropy solution of (1.1). Note that Lemma 6.1 ensures the boundedness of $u$ in $L^{\infty}\left(G_{T}\right)$.

\section{Uniqueness for the conservation law}

In this section we are going to prove uniqueness of entropy solutions (see Theorem 9.4) as defined in Definition 4.3 or 4.5. For the sake of brevity we suppress the integration elements $d x, d y, d t$ and $d \tau$ the in this section. Integration is meant to be done over each of these variables that occur in the respective integral.

We will need that the initial data is approached in the following sense, which is a fine property of scalar conversation laws. Related analysis considering divergence measure fields and its applications to conservation laws posed in the Euclidean setting can be found in [10-12].

LEMMA 9.1 An entropy solution $u=u(x, t)$ approaches its initial values in the following sense.

$$
\underset{t \searrow 0}{\operatorname{ess} \lim } \int_{\Gamma_{0}}\left|u(\Phi(x, t), t)-u_{0}(x)\right|^{2}=0 .
$$

Proof. Recall that an entropy solution is a weak solution. Hence we use (4.1) with $\varphi(x, t):=$ $\theta_{\epsilon, \tilde{\epsilon}}(t) \chi\left((\Phi(\cdot, t))^{-1}(x)\right)$, where

$$
\theta_{\epsilon, \tilde{\epsilon}}(t):=\left\{\begin{array}{cl}
1 & \text { for } 0 \leqslant t \leqslant \epsilon \\
1-(t-\epsilon) / \tilde{\epsilon} & \text { for } \epsilon<t \leqslant \epsilon+\tilde{\epsilon} \\
0 & \text { else }
\end{array}\right.
$$

and $\chi \in C^{\infty}\left(\Gamma_{0}\right)$ is arbitrary in order to obtain for $\tilde{\epsilon} \searrow 0$ and almost every $\epsilon$

$$
-\int_{\Gamma(\epsilon)} u(x, \epsilon) \chi\left((\Phi(\cdot, \epsilon))^{-1}(x)\right)+\int_{\Gamma_{0}} u_{0}(x) \chi(x)=\mathcal{O}(\epsilon) .
$$


Invoking the density of $C^{\infty}\left(\Gamma_{0}\right)$ in $L^{1}\left(\Gamma_{0}\right)$ we see that (9.2) is even true for $\chi \in L^{1}\left(\Gamma_{0}\right)$. Similarly, by choosing $\phi(x, t):=\theta_{\epsilon, \tilde{\epsilon}}(t)$ as a test function in (4.9) we get for almost every $\epsilon$

$$
-\int_{\Gamma_{0}} \eta\left(u_{0}(x)\right)+\int_{\Gamma(\epsilon)} \eta(u(x, \epsilon)) \leqslant \mathcal{O}(\epsilon) .
$$

A combination of (9.2) and (9.3) yields

$$
\begin{aligned}
\operatorname{ess} \limsup _{\epsilon \searrow 0} \int_{\Gamma_{0}} \eta(u(\Phi(x, \epsilon), \epsilon)) \mid \operatorname{det} & D \Phi(x, \epsilon) \mid-\eta\left(u_{0}(x)\right) \\
& -\eta^{\prime}\left(u_{0}(x)\right)\left(u(\Phi(x, \epsilon), \epsilon)|\operatorname{det} D \Phi(x, \epsilon)|-u_{0}(x)\right) \leqslant 0 .
\end{aligned}
$$

By choosing $\eta(u)=u^{2}$ and using the fact that $|\operatorname{det} D \Phi(x, \epsilon)|=1+\mathcal{O}(\epsilon)$ the proof is completed.

For the proof of uniqueness we need some technical definitions and basic facts from differential geometry. For a parametrization $\tilde{\psi}: U \rightarrow \tilde{\psi}(U)$ of a subset $\tilde{\psi}(U) \subset \Gamma_{0}$ with $U \subset \mathbb{R}^{n}$ open we have the following properties.

(a) For $t>0$ a parametrization of $\Phi(\tilde{\psi}(U), t) \subset \Gamma(t)$ is given by the map $\psi(\cdot, t): U \rightarrow \psi(U)$ where

$$
\psi(x, t):=\Phi(\tilde{\psi}(x), t) .
$$

(b) For a function $\varphi \in C^{1}\left(G_{T}\right)$ the material derivative has the local form

$$
\dot{\varphi}(\psi(x, t), t)=(\varphi(\psi(x, t), t))_{t} .
$$

(c) For the local representation $W=W_{i}\left(\psi_{x_{i}}(\cdot, t) \circ \psi(\cdot, t)^{-1}\right)$ of a tangential vector field $W$ on $\Gamma(t)$ we have

$$
\left(W \cdot \nabla_{\Gamma(t)} \varphi\right) \circ \psi(\cdot, t)=\left(W_{i} \circ \psi(t, \cdot)\right)(\varphi \circ \psi(\cdot, t))_{x_{i}}
$$

for all $\varphi \in C^{1}(\Gamma(t))$.

(d) For any function $\alpha \in L^{1}(\Gamma(t))$ we have the following local computation of the integral over a subset $\psi(V, t) \subset \Gamma(t)$ where $V \subset U$.

$$
\int_{\psi(V, t)} \alpha=\int_{V} \alpha \circ \psi(\cdot, t) \sqrt{\operatorname{det}\left(\mathrm{D} \psi(\cdot, t)^{T} \mathrm{D} \psi(\cdot, t)\right)} .
$$

Here $\mathrm{D}$ denotes the Jacobian operator w.r.t. the spatial coordinate $x \in U \subset \mathbb{R}^{n}$.

We introduce a function $\delta \in C^{\infty}(\mathbb{R})$ satisfying $\delta \geqslant 0$, supp $\delta \subset[-1,1]$ and $\int_{\mathbb{R}} \delta(\sigma) d \sigma=1$. For $h>0$ we set $\delta_{h}(\sigma):=\frac{1}{h} \delta\left(\frac{\sigma}{h}\right)$, then $\delta_{h} \in C^{\infty}(\mathbb{R})$, supp $\delta_{h} \subset[-h, h], \delta_{h}^{\prime} \leqslant \frac{C}{h}$ and $\int_{\mathbb{R}} \delta_{h}(\sigma) d \sigma=1$ for some constant $C$. We need the following two Lemmata whose proofs can be found in [25].

LEMMA 9.2 If a function $F(u)$ satisfies a Lipschitz condition on an interval $[-M, M]$ with constant $L$, then the function $H(u, \bar{u}):=\operatorname{sign}(u-\bar{u})[F(u)-F(\bar{u})]$ also satisfies the Lipschitz condition in $u$ and $\bar{u}$ with the constant $L$. 
LEMma 9.3 Let the function $\bar{u}(x, t)$ be bounded and measurable in some cylinder $B_{r}(0) \times[0, T]$. If for some $\rho \in(0, \min (r, T))$ and any number $h \in(0, \rho)$ we set

$$
V_{h}:=\frac{1}{h^{n+1}} \iiint \int_{\substack{\left|\frac{t-\tau}{2}\right| \leqslant h, \rho \leqslant \frac{t+\tau}{2} \leqslant T-\rho,\left\|\frac{x-y}{2}\right\| \leqslant h,\left\|\frac{x+y}{2}\right\| \leqslant r-\rho}}|\bar{u}(x, t)-\bar{u}(y, \tau)| d x d t d y d \tau,
$$

then $\lim _{h \rightarrow 0} V_{h}=0$.

THEOREM 9.4 Assume Assumptions 2.1 and $\nabla_{\Gamma(t)} \cdot v \in L^{\infty}\left(G_{T}\right)$ and $\dot{f}$ and $\nabla_{\Gamma(t)} f$ are Lipschitz continuous with respect to $u$. Then the Kruzkov entropy solution of Definition 4.5 is unique.

Proof. The proof we give here is in the same spirit as Kruzkov's uniqueness proof [25] and can be seen as its extension to moving surfaces. Let $u, \bar{u}$ be two Kruzkov entropy solutions with initial data $u_{0}, \bar{u}_{0}$. Furthermore let $\psi$ be as in (9.4) with $\psi(\cdot, t): B_{R}(0) \rightarrow \psi\left(B_{R}(0), t\right)$ where $B_{R}(0) \subset \mathbb{R}^{n}$ and $\psi\left(B_{R}(0), t\right) \subset \Gamma(t)$. Choose normal coordinates for instance. For some $0<r<R$ we define $\Omega:=B_{r}(0)$ and $\Omega_{T}:=B_{r}(0) \times[0, T]$. Let now $\varphi \in C^{\infty}\left(G_{T}\right)$ be a test function with $\varphi \geqslant 0$ and supp $\varphi \Subset \bigcup_{t \in(\rho, T-2 \rho)} \psi(\Omega, t) \times\{t\}$ where $\rho>0$ is a small real number. We know because of (a)-(d) that then

$$
\begin{aligned}
& \int_{G_{T}}|u(x, t)-k| \dot{\varphi}-\operatorname{sign}(u(x, t)-k) k \nabla_{\Gamma(t)} \cdot v(x, t) \varphi(x, t) \\
& +\operatorname{sign}(u(x, t)-k)(f(x, t, u(x, t))-f(x, t, k)) \cdot \nabla_{\Gamma(t)} \varphi(x, t)+\int_{\Gamma_{0}}\left|u_{0}(x)-k\right| \varphi(x, 0) \\
& =\int_{0}^{T} \int_{\Omega}\left[| u ( \psi ( x , t ) , t ) - k | \left(\varphi(\psi(x, t), t)_{t}\right.\right. \\
& -\operatorname{sign}(u(\psi(x, t), t)-k) k\left(\nabla_{\Gamma(t)} \cdot v\right)(\psi(x, t), t) \varphi(\psi(x, t), t)+\operatorname{sign}(u(\psi(x, t), t)-k) \\
& \left.\quad \cdot\left(f_{i}(\psi(x, t), t, u(\psi(x, t), t))-f_{i}(\psi(x, t), t, k)\right)(\varphi(\psi(x, t), t))_{x_{i}}\right] \sqrt{\operatorname{det}(g(x, t))}
\end{aligned}
$$

where $f_{i}$ is locally defined by $f=f_{i} \cdot\left(\psi_{x_{i}}(\cdot, t) \circ \psi(\cdot, t)^{-1}\right)$ and $g(x, t):=\mathrm{D} \psi(x, t)^{T} \mathrm{D} \psi(x, t)$, where $\mathrm{D} \psi(x, t)$ denotes the Jacobian of $\psi(x, t)$ with respect to $x$. For better readability we will suppress the composition with $\psi(\cdot, t)$ in the following, i.e. we introduce new functions which live on $\Omega \times[0, T]$ and which we mainly again denote by the names of the original functions. By this we mean to do the following replacements. $u(\psi(x, t), t) \rightsquigarrow u(x, t), \varphi(\psi(x, t), t) \rightsquigarrow \varphi(x, t)$, $\left(\nabla_{\Gamma(t)} \cdot v\right)(\psi(x, t), t) \rightsquigarrow q(x, t)$ and $f_{i}(\psi(x, t), t, \cdot) \rightsquigarrow f_{i}(x, t, \cdot)$.

In Definition 4.5 we choose a test function

$$
\tilde{\varphi}=\tilde{\varphi}(x, t, y, \tau) \geqslant 0 \quad \text { with } \quad \operatorname{supp} \tilde{\varphi} \Subset\left(\bigcup_{t \in(\rho, T-2 \rho)} \psi(\Omega, t) \times\{t\}\right)^{2},
$$

set $k=\bar{u}(y, \tau)$, multiply with $\sqrt{\operatorname{det}(g(y, \tau))}$ and integrate over $\Omega_{T}:=\Omega \times[0, T]$ with respect to 
$(y, \tau)$. Using $(9.9)$ we arrive at

$$
\begin{gathered}
\int_{\Omega_{T}^{2}}\left[|u(x, t)-\bar{u}(y, \tau)| \tilde{\varphi}_{t}(x, t, y, \tau)-\operatorname{sign}(u(x, t)-\bar{u}(y, \tau)) \bar{u}(y, \tau) q(x, t) \tilde{\varphi}(x, t, y, \tau)\right. \\
\left.+\operatorname{sign}(u(x, t)-\bar{u}(y, \tau))\left(f_{i}(x, t, u(x, t))-f_{i}(x, t, \bar{u}(y, \tau))\right) \tilde{\varphi}_{x_{i}}(x, t, y, \tau)\right] \\
\sqrt{\operatorname{det}(g(x, t))} \sqrt{\operatorname{det}(g(y, \tau))} \geqslant 0 .
\end{gathered}
$$

Proceeding analogously for the corresponding version of (4.10) for the entropy solution $\bar{u}=\bar{u}(y, \tau)$ we get

$$
\begin{gathered}
\int_{\Omega_{T}^{2}}\left[|u(x, t)-\bar{u}(y, \tau)| \tilde{\varphi}_{\tau}(x, t, y, \tau)+\operatorname{sign}(u(x, t)-\bar{u}(y, \tau)) u(x, t) q(y, \tau) \tilde{\varphi}(x, t, y, \tau)\right. \\
\left.+\operatorname{sign}(u(x, t)-\bar{u}(y, \tau))\left(f_{i}(y, \tau, u(x, t))-f_{i}(y, \tau, \bar{u}(y, \tau))\right) \tilde{\varphi}_{y_{i}}(x, t, y, \tau)\right] \\
\sqrt{\operatorname{det}(g(x, t))} \sqrt{\operatorname{det}(g(y, \tau))} \geqslant 0 .
\end{gathered}
$$

Summing up (9.10) and (9.11) one sees

$$
\begin{gathered}
\int_{\Omega_{T}^{2}}\left[|u(x, t)-\bar{u}(y, \tau)|\left(\tilde{\varphi}_{t}(x, t, y, \tau)+\tilde{\varphi}_{\tau}(x, t, y, \tau)\right)\right. \\
+\operatorname{sign}(u(x, t)-\bar{u}(y, \tau))\left(f_{i}(x, t, u(x, t))-f_{i}(y, \tau, \bar{u}(y, \tau))\right)\left(\tilde{\varphi}_{x_{i}}(x, t, y, \tau)+\tilde{\varphi}_{y_{i}}(x, t, y, \tau)\right) \\
+\underbrace{\operatorname{sign}(u(x, t)-\bar{u}(y, \tau))\left(f_{i}(y, \tau, \bar{u}(y, \tau))-f_{i}(x, t, \bar{u}(y, \tau))\right) \tilde{\varphi}_{x_{i}}(x, t, y, \tau)}_{=: R_{1}} \\
+\underbrace{\operatorname{sign}(u(x, t)-\bar{u}(y, \tau))\left(f_{i}(y, \tau, u(x, t))-f_{i}(x, t, u(x, t))\right) \tilde{\varphi}_{y_{i}}(x, t, y, \tau)}_{=: R_{2}} \\
\quad+\underbrace{\operatorname{sign}(u(x, t)-\bar{u}(y, \tau))(u(x, t) q(y, \tau)-\bar{u}(y, \tau) q(x, t))}_{=: Q} \tilde{\varphi}(x, t, y, \tau)] \\
\sqrt{\operatorname{det}(g(x, t))} \sqrt{\operatorname{det}(g(y, \tau))} \geqslant 0 . \quad \text { (9.12) }
\end{gathered}
$$

For a test function $\varphi=\varphi(x, t) \geqslant 0$ satisfying supp $\varphi \Subset \bigcup_{t \in(\rho, T-2 \rho)} \psi(\Omega, t) \times\{t\}$ we set (in local coordinates)

$$
\tilde{\varphi}(x, t, y, \tau):=\varphi\left(\frac{x+y}{2}, \frac{t+\tau}{2}\right) \delta_{h}\left(\frac{t-\tau}{2}\right) \prod_{i=1}^{n} \delta_{h}\left(\frac{x_{i}-y_{i}}{2}\right)=: \varphi(\cdots) \lambda_{h}(\vdots)
$$

where

$$
(\cdots)=\left(\frac{x+y}{2}, \frac{t+\tau}{2}\right), \quad(\vdots)=\left(\frac{x-y}{2}, \frac{t-\tau}{2}\right)
$$


and $h$ is sufficiently small, such that supp $\tilde{\varphi} \Subset\left(\bigcup_{t \in(\rho, T-2 \rho)} \psi(\Omega, t) \times\{t\}\right)^{2}$. For the partial derivatives of $\tilde{\varphi}$ the following identities are trivial.

$$
\begin{gathered}
\tilde{\varphi}_{t}+\tilde{\varphi}_{\tau}=\varphi_{t}(\cdots) \lambda_{h}(\vdots), \\
\tilde{\varphi}_{x_{i}}+\tilde{\varphi}_{y_{i}}=\varphi_{x_{i}}(\cdots) \lambda_{h}(\vdots) .
\end{gathered}
$$

The major part of the proof will be to see that with $\tilde{\varphi}$ as in (9.13) the following inequality is obtained from (9.12) for $h \rightarrow 0$ :

$$
\begin{aligned}
\int_{\Omega_{T}} & {\left[|u(x, t)-\bar{u}(x, t)| \varphi_{t}(x, t)\right.} \\
& +\operatorname{sign}(u(x, t)-\bar{u}(x, t))\left(f_{i}(x, t, u(x, t))-f_{i}(x, t, \bar{u}(x, t))\right) \varphi_{x_{i}}(x, t) \\
& -\operatorname{sign}(u(x, t)-\bar{u}(x, t))\left(f_{i_{x_{i}}}(x, t, u(x, t))-f_{i_{x_{i}}}(x, t, \bar{u}(x, t))\right) \varphi(x, t) \\
& +|u(x, t)-\bar{u}(x, t)| q(x, t) \varphi(x, t)] \operatorname{det}(g(x, t)) \geqslant 0 .
\end{aligned}
$$

In order to prove (9.16) we define a function

$$
\begin{aligned}
& F(x, t, y, \tau, u(x, t), \bar{u}(y, \tau)) \lambda_{h}(\vdots) \\
&:=\left[|u(x, t)-\bar{u}(y, \tau)| \varphi_{t}(\cdots)\right. \\
&+\operatorname{sign}(u(x, t)-\bar{u}(y, \tau))\left(f_{i}(x, t, u(x, t))-f_{i}(y, \tau, \bar{u}(y, \tau))\right) \varphi_{x_{i}}(\cdots) \\
&+Q \varphi(\cdots)] \sqrt{\operatorname{det}(g(x, t))} \sqrt{\operatorname{det}(g(y, \tau))} \lambda_{h}(\vdots) .
\end{aligned}
$$

From (9.12) we deduce

$$
\begin{aligned}
0 \leqslant & \int_{\Omega_{T}^{2}} F(x, t, y, \tau, u(x, t), \bar{u}(y, \tau)) \lambda_{h}(\vdots) \\
& +\int_{\Omega_{T}^{2}}\left[R_{1}+R_{2}\right] \sqrt{\operatorname{det}(g(x, t))} \sqrt{\operatorname{det}(g(y, \tau))} \\
= & \int_{\Omega_{T}^{2}}[F(x, t, y, \tau, u(x, t), \bar{u}(y, \tau))-F(x, t, x, t, u(x, t), \bar{u}(x, t))] \lambda_{h}(\vdots) \\
& +\int_{\Omega_{T}^{2}} F(x, t, x, t, u(x, t), \bar{u}(x, t)) \lambda_{h}(\vdots) \\
& +\int_{\Omega_{T}^{2}}\left[R_{1}+R_{2}\right] \sqrt{\operatorname{det}(g(x, t))} \sqrt{\operatorname{det}(g(y, \tau))}=: J_{1}(h)+J_{2}+J_{3}(h) .
\end{aligned}
$$

We mention that due to Lemma 9.2 and since $\bar{\Omega}_{T} \subset B_{R}(0) \times[0, T]$ and $F$ is defined on $\left(B_{R}(0) \times\right.$ $[0, T])^{2}$, obviously $F$ is Lipschitz continuous on $\left(\Omega_{T}\right)^{2}$ in all its arguments. We then use the fact 
that $\left|\lambda_{h}(\vdots)\right| \leqslant C h^{-(n+1)}$ and Lemma 9.3 to see that

$$
\left|J_{1}(h)\right| \leqslant C\left(h+\int_{\Omega_{T}^{2}}|\bar{u}(y, \tau)-\bar{u}(x, t)| \lambda_{h}(\vdots)\right) \rightarrow 0
$$

for $h \rightarrow 0$ since $\bar{u}$ is bounded and measurable. By substitution we get for the second term

$$
\begin{aligned}
J_{2} & =\int_{\Omega_{T}} F(x, t, x, t, u(x, t), \bar{u}(x, t)) \underbrace{\int_{\Omega_{T}} \lambda_{h}\left(\frac{x-y}{2}, \frac{t-\tau}{2}\right)}_{=2^{n+1}} \\
& =2^{n+1} \int_{\Omega_{T}} F(x, t, x, t, u(x, t), \bar{u}(x, t)) d x d t .
\end{aligned}
$$

Now we turn to the third term

$$
\begin{gathered}
J_{3}(h)=\int_{\Omega_{T}^{2}}\left[\operatorname{sign}(u(x, t)-\bar{u}(y, \tau))\left(f_{i}(y, \tau, \bar{u}(y, \tau))-f_{i}(x, t, \bar{u}(y, \tau))\right)\right. \\
\cdot\left(\frac{1}{2} \varphi_{x_{i}}(\cdots) \lambda_{h}(\vdots)+\varphi(\cdots)\left(\lambda_{h}(\vdots)\right)_{x_{i}}\right) \\
+\operatorname{sign}(u(x, t)-\bar{u}(y, \tau))\left(f_{i}(y, \tau, u(x, t))-f_{i}(x, t, u(x, t))\right) \\
\left.\cdot\left(\frac{1}{2} \varphi_{x_{i}}(\cdots) \lambda_{h}(\vdots)+\varphi(\cdots)\left(\lambda_{h}(\vdots)\right)_{y_{i}}\right)\right] \\
\sqrt{\operatorname{det}(g(x, t))} \sqrt{\operatorname{det}(g(y, \tau))} .
\end{gathered}
$$

We notice that those summands of the above integral that contain $\varphi_{x_{i}}(\cdots) \lambda_{h}(\vdots)$ as a factor in the integrand vanish for $h \rightarrow 0$. Consequently, it suffices to show that

$$
\begin{gathered}
\hat{J}_{3}(h):=\int_{\Omega_{T}^{2}} \operatorname{sign}(u(x, t)-\bar{u}(y, \tau))\left[\left(f_{i}(y, \tau, \bar{u}(y, \tau))-f_{i}(x, t, \bar{u}(y, \tau))\right)\left(\lambda_{h}(\vdots)\right)_{x_{i}}\right. \\
\left.+\left(f_{i}(y, \tau, u(x, t))-f_{i}(x, t, u(x, t))\right)\left(\lambda_{h}(\vdots)\right)_{y_{i}}\right] \hat{\varphi}(x, t, y, \tau)
\end{gathered}
$$

converges to

$$
-2^{n+1} \int_{\Omega_{T}} \operatorname{sign}(u(x, t)-\bar{u}(x, t))\left(f_{i_{x_{i}}}(x, t, u(x, t))-f_{i_{x_{i}}}(x, t, \bar{u}(x, t))\right) \varphi(x, t) \operatorname{det}(g(x, t))
$$

for $h \rightarrow 0$, where $\hat{\varphi}(x, t, y, \tau):=\varphi(\cdots) \sqrt{\operatorname{det}(g(x, t))} \sqrt{\operatorname{det}(g(y, \tau))}$. For a better readability we write $\lambda_{x_{i}}$ instead of $\left(\lambda_{h}(\vdots)\right)_{x_{i}}$ and analogously $\lambda_{y_{i}}$. Due to the local Lipschitz continuity of $f_{i}$ on $\Omega_{T}$ we have

$$
\begin{aligned}
\left(f_{i}(y, \tau, \bar{u}(y, \tau))-f_{i}(x, t, \bar{u}(y, \tau))\right) \lambda_{x_{i}}= & f_{i_{\tau}}(y, \tau, \bar{u}(y, \tau))(\tau-t) \lambda_{x_{i}} \\
& +f_{i_{y_{j}}}(y, \tau, \bar{u}(y, \tau))\left(y_{j}-x_{j}\right) \lambda_{x_{i}}+\epsilon_{i} \lambda_{x_{i}}
\end{aligned}
$$


with $\frac{\epsilon_{i}}{d} \rightarrow 0$ for $d:=\|x-y\|+|t-\tau| \rightarrow 0$ where $\|\cdot\|$ denotes the Euclidean norm in $\mathbb{R}^{n}$. Analogously, with $\lambda_{x_{i}}=-\lambda_{y_{i}}$ we get

$$
\begin{aligned}
\left(f_{i}(y, \tau, u(x, t))-f_{i}(x, t, u(x, t))\right) \lambda_{y_{i}}= & -f_{i_{\tau}}(y, \tau, u(x, t))(\tau-t) \lambda_{x_{i}} \\
& -f_{i_{y_{j}}}(y, \tau, u(x, t))\left(y_{j}-x_{j}\right) \lambda_{x_{i}}+\beta_{i} \lambda_{x_{i}}
\end{aligned}
$$

with $\frac{\beta_{i}}{d} \rightarrow 0$ for $d \rightarrow 0$. Thus,

$$
\begin{gathered}
\hat{J}_{3}(h)=\int_{\Omega_{T}^{2}} \hat{\varphi}(x, t, y, \tau) \operatorname{sign}(u(x, t)-\bar{u}(y, \tau))\left(f_{i_{\tau}}(y, \tau, \bar{u}(y, \tau))-f_{i_{\tau}}(y, \tau, u(x, t))\right)(\tau-t) \lambda_{x_{i}} \\
+\int_{\Omega_{T}^{2}} \hat{\varphi}(x, t, y, \tau) \operatorname{sign}(u(x, t)-\bar{u}(y, \tau))\left(f_{i_{y_{j}}}(y, \tau, \bar{u}(y, \tau))-f_{i_{y_{j}}}(y, \tau, u(x, t))\right)\left(y_{j}-x_{j}\right) \lambda_{x_{i}} \\
\quad+\int_{\Omega_{T}^{2}} \hat{\varphi}(x, t, y, \tau) \operatorname{sign}(u(x, t)-\bar{u}(y, \tau))\left(\epsilon_{i}+\beta_{i}\right) \lambda_{x_{i}}=: I_{1}+I_{2}+I_{3} .
\end{gathered}
$$

Obviously, $\left|\lambda_{x_{i}}\right| \leqslant C h^{-(n+2)}$ and using Lemma 9.3 we get $\left|I_{3}\right| \rightarrow 0$ as $h \rightarrow 0$. Since $\sqrt{\operatorname{det}(g)}$ is Lipschitz continuous on $\Omega_{T}$ we have

$$
|\hat{\varphi}(x, t, y, \tau)-\hat{\varphi}(x, t, x, t)| \leqslant C(\|x-y\|+|t-\tau|)
$$

and obtain

$$
\begin{aligned}
I_{1}=\int_{\Omega_{T}^{2}}[\hat{\varphi}(y, \tau, y, \tau) \operatorname{sign}(u(x, t)-\bar{u}(y, \tau)) \\
\left.\quad\left(f_{i_{\tau}}(y, \tau, \bar{u}(y, \tau))-f_{i_{\tau}}(y, \tau, u(x, t))\right)(\tau-t) \lambda_{x_{i}}\right]+\gamma_{1}(h)
\end{aligned}
$$

with $\gamma_{1}(h) \rightarrow 0$ for $h \rightarrow 0$. Defining

$$
\begin{array}{r}
F_{i}(y, \tau, u(x, t), \bar{u}(y, \tau)):=\hat{\varphi}(y, \tau, y, \tau) \operatorname{sign}(u(x, t)-\bar{u}(y, \tau)) \\
\left(f_{i_{\tau}}(y, \tau, \bar{u}(y, \tau))-f_{i_{\tau}}(y, \tau, u(x, t))\right)
\end{array}
$$

we see with Lemma 9.2 that $F_{i}$ is Lipschitz continuous in $u$ on $\Omega_{T}$ and obtain according to Lemma 9.3

$$
\begin{aligned}
\left|I_{1}\right| & \leqslant\left|\int_{\Omega_{T}^{2}} F_{i}(y, \tau, u(x, t), \bar{u}(y, \tau))((\tau-t) \lambda)_{x_{i}}\right|+\left|\gamma_{1}(h)\right| \\
& =\left|\int_{\Omega_{T}^{2}}\left(F_{i}(y, \tau, u(x, t), \bar{u}(y, \tau))-F_{i}(y, \tau, u(y, \tau), \bar{u}(y, \tau))\right)((\tau-t) \lambda)_{x_{i}}\right|+\left|\gamma_{1}(h)\right| \\
& \leqslant C \int_{\Omega_{T}^{2}}|u(x, t)-u(y, \tau)| \underbrace{|(\tau-t)|}_{\leqslant h} \underbrace{\left|\lambda_{x_{i}}\right|}_{\leqslant C h^{-(n+2)}}+\left|\gamma_{1}(h)\right| \rightarrow 0
\end{aligned}
$$


for $h \rightarrow 0$. Considering $I_{2}$ we have

$$
\begin{array}{r}
I_{2}=\int_{\Omega_{T}^{2}} \hat{\varphi}(x, t, y, \tau) \operatorname{sign}(u(x, t)-\bar{u}(y, \tau)) \\
\left(f_{i_{y_{j}}}(y, \tau, \bar{u}(y, \tau))-f_{i_{y_{j}}}(y, \tau, u(x, t))\right)\left(\left(y_{j}-x_{j}\right) \lambda\right)_{x_{i}} \\
+\int_{\Omega_{T}^{2}} \hat{\varphi}(x, t, y, \tau) \operatorname{sign}(u(x, t)-\bar{u}(y, \tau))\left(f_{i_{y_{i}}}(y, \tau, \bar{u}(y, \tau))-f_{i_{y_{i}}}(y, \tau, u(x, t))\right) \lambda \\
=: I_{2.1}+I_{2.2} .
\end{array}
$$

We see that $I_{2.2}$ converges for $h \rightarrow 0$ to

$$
2^{n+1} \int_{\Omega_{T}} \operatorname{sign}(u(x, t)-\bar{u}(x, t))\left(f_{i_{x_{i}}}(x, t, \bar{u}(x, t))-f_{i_{x_{i}}}(x, t, u(x, t))\right) \varphi(x, t) \operatorname{det}(g(x, t)),
$$

whereas one can see analogously to $I_{1}$ that $I_{2.1}$ converges to zero. Thus, we conclude that

$$
\begin{aligned}
& 0 \leqslant \lim _{h \rightarrow 0}\left[J_{1}(h)+J_{2}+J_{3}(h)\right]=2^{n+1} \int_{\Omega_{T}} F(x, t, x, t, u(x, t), \bar{u}(x, t)) \\
& -2^{n+1} \int_{\Omega_{T}} \operatorname{sign}(u(x, t)-\bar{u}(x, t))\left(f_{i_{i}}(x, t, u(x, t))-f_{i_{x_{i}}}(x, t, \bar{u}(x, t))\right) \varphi(x, t) \operatorname{det}(g(x, t))
\end{aligned}
$$

and thereby (9.16).

In order to continue the proof of the theorem we introduce the following definitions. Let $\varepsilon_{u} \subset$ $[0, T]$ be of $\mathcal{L}^{1}$-measure zero, such that $u(\Phi(\cdot, t), t) \rightarrow u_{0}$ in $L^{1}\left(\Gamma_{0}\right)$ for $t \rightarrow 0, t \in[0, T] \backslash \varepsilon_{u}$. Let $\varepsilon_{\bar{u}}$ be defined analogously. These sets exist because of Lemma 9.1. We set

$$
\mu(t):=\int_{S_{t}}|u(x, t)-\bar{u}(x, t)| \operatorname{det}(g(x, t))
$$

where $S_{t}:=\left\{x \in B_{r}(0) \mid\|x\| \leqslant r-L t\right\}$ and

$$
L:=\max _{\substack{(x, t) \in \bar{\Omega}_{T}, \bar{\Omega} \\ w \leqslant \max \left(\|u\|_{L} \infty,\|\bar{u}\|_{L}\right)}}\left(\sum_{i=1}^{n} \partial_{u} f_{i}^{2}(x, t, w)\right)^{\frac{1}{2}}
$$

By $\varepsilon_{\mu} \subset[0, T]$ we denote those points that are not Lebesgue points of the bounded and measurable function $\mu$ and set $\varepsilon_{0}:=\varepsilon_{u} \cup \varepsilon_{\bar{u}} \cup \varepsilon_{\mu}$. Obviously, $\mathcal{L}^{1}\left(\varepsilon_{0}\right)=0$. We define

$$
a_{h}(\sigma):=\int_{-\infty}^{\sigma} \delta_{h}(\xi) d \xi
$$

as a regularization of the Heavyside function and see $a_{h}^{\prime}(\sigma)=\delta_{h}(\sigma) \geqslant 0$. Let now $\tau_{1}, \tau_{2} \in$ $(0, T) \backslash \varepsilon_{0}$ with $\tau_{1}<\tau_{2}$. In order to prove a contraction property we choose the following test function whose definition is given in local coordinates by

$$
\varphi(x, t):=\left[a_{h}\left(t-\tau_{1}\right)-a_{h}\left(t-\tau_{2}\right)\right] \zeta(x, t),
$$


where

$$
\zeta(x, t):=\zeta_{\epsilon}(x, t):=1-a_{\epsilon}(\|x\|+L t-r+\epsilon), \quad \epsilon>0
$$

for $(x, t) \in \Omega_{T}$ and zero outside. Hence, $\operatorname{supp} \zeta(\cdot, t) \subset S_{t} \subset B_{r}(0)$. We compute the derivatives of $\zeta$ as

$$
\zeta_{t}(x, t):=-L \underbrace{a_{\epsilon}^{\prime}(\|x\|+L t-r+\epsilon)}_{\geqslant 0} \text { and } \nabla \zeta(x, t):=-a_{\epsilon}^{\prime}(\|x\|+L t-r+\epsilon) \frac{x}{\|x\|}
$$

and due to the definition of $L$ we conclude with the Cauchy Schwartz inequality

$$
0=\zeta_{t}(x, t)+L\|\nabla \zeta(x, t)\| \geqslant \zeta_{t}(x, t)+\frac{f_{i}(x, t, u(x, t))-f_{i}(x, t, \bar{u}(x, t))}{u(x, t)-\bar{u}(x, t)} \zeta_{x_{i}}(x, t) .
$$

If we choose the function $\varphi$ from (9.37) as a test function in (9.16) we get

$$
\begin{gathered}
\int_{\Omega_{T}}\left[\delta_{h}\left(t-\tau_{1}\right)-\delta_{h}\left(t-\tau_{2}\right)\right] \zeta(x, t)|u(x, t)-\bar{u}(x, t)| \operatorname{det}(g(x, t)) \\
+\int_{\Omega_{T}} \underbrace{\left[a_{h}\left(t-\tau_{1}\right)-a_{h}\left(t-\tau_{2}\right)\right]}_{\geqslant 0} \underbrace{|u(x, t)-\bar{u}(x, t)|}_{\geqslant 0} \\
\underbrace{\left[\zeta_{t}(x, t)+\frac{f_{i}(x, t, u(x, t))-f_{i}(x, t, \bar{u}(x, t))}{u(x, t)-\bar{u}(x, t)} \zeta_{x_{i}}(x, t)\right]}_{\leqslant 0} \underbrace{\operatorname{det}(g(x, t))}_{>0} \\
-\int_{\Omega_{T} \quad \operatorname{sign}(u(x, t)-\bar{u}(x, t))\left(f_{i x_{i}}(x, t, u(x, t))-f_{i x_{i}}(x, t, \bar{u}(x, t))\right)}^{\left(g_{1}\left(t-\tau_{1}\right)-a_{h}\left(t-\tau_{2}\right)\right] \zeta(x, t) \operatorname{det}(g(x, t))} \\
+\int_{\Omega_{T}}|u(x, t)-\bar{u}(x, t)| q(x, t)\left[a_{h}\left(t-\tau_{1}\right)-a_{h}\left(t-\tau_{2}\right)\right] \zeta(x, t) \operatorname{det}(g(x, t)) \geqslant 0 .
\end{gathered}
$$

For $\epsilon \rightarrow 0$ we have

$$
\begin{gathered}
\int_{0}^{T}\left[\delta_{h}\left(t-\tau_{1}\right)-\delta_{h}\left(t-\tau_{2}\right)\right] \int_{S_{t}}|u(x, t)-\bar{u}(x, t)| \operatorname{det}(g(x, t)) \\
-\int_{0}^{T}\left[a_{h}\left(t-\tau_{1}\right)-a_{h}\left(t-\tau_{2}\right)\right] \int_{S_{t}} \operatorname{sign}(u(x, t)-\bar{u}(x, t)) \\
\quad \cdot\left(f_{i x_{i}}(x, t, u(x, t))-f_{i x_{i}}(x, t, \bar{u}(x, t))\right) \operatorname{det}(g(x, t)) \\
+\int_{0}^{T}\left[a_{h}\left(t-\tau_{1}\right)-a_{h}\left(t-\tau_{2}\right)\right] \int_{S_{t}}|u(x, t)-\bar{u}(x, t)| q(x, t) \operatorname{det}(g(x, t)) \geqslant 0 .
\end{gathered}
$$


If now $h \rightarrow 0$ this implies

$$
\begin{aligned}
\mu\left(\tau_{2}\right)= & \int_{S_{\tau_{2}}}\left|u\left(x, \tau_{2}\right)-\bar{u}\left(x, \tau_{2}\right)\right| \operatorname{det}\left(g\left(x, \tau_{2}\right)\right) \\
\leqslant & \int_{S_{\tau_{1}}}\left|u\left(x, \tau_{1}\right)-\bar{u}\left(x, \tau_{1}\right)\right| \operatorname{det}\left(g\left(x, \tau_{1}\right)\right) \\
& \quad+\int_{\tau_{1}}^{\tau_{2}} \int_{S_{t}}|u(x, t)-\bar{u}(x, t)|\left(|q(x, t)|+L\left(f_{i_{x_{i}}}\right)\right) \operatorname{det}(g(x, t)) \\
\leqslant & \mu\left(\tau_{1}\right)+\left(\|q\|_{L^{\infty}}+L\left(f_{i_{x_{i}}}\right)\right) \int_{\tau_{1}}^{\tau_{2}} \mu(t),
\end{aligned}
$$

where $L\left(f_{i_{x_{i}}}\right)$ denotes the local Lipschitz constant of $f_{i_{x_{i}}}$ on $\Omega_{T}$ and by a Gronwall argument we conclude

$$
\mu\left(\tau_{2}\right) \leqslant \mu\left(\tau_{1}\right) \exp \left(\left(\|q\|_{L^{\infty}}+L\left(f_{i_{i}}\right)\right)\left(\tau_{2}-\tau_{1}\right)\right) .
$$

Using the fact that

$$
|| u-\bar{u}|-| u_{0}-\bar{u}_{0}|| \leqslant\left|u_{0}-u\right|+\left|\bar{u}_{0}-\bar{u}\right|
$$

and that $\operatorname{det}(g(x, t))$ is bounded and Lipschitz in $t$ we get with Lemma 9.1 for $\tau_{1} \rightarrow 0$ in $[0, T] \backslash \varepsilon_{0}$ the following estimate

$$
\mu\left(\tau_{2}\right) \leqslant \exp \left(\left(\|q\|_{L^{\infty}}+L\left(f_{i_{x_{i}}}\right)\right) \tau_{2}\right) \int_{B_{r}(0)}\left|u_{0}(x)-\bar{u}_{0}(x)\right| \operatorname{det}(g(x, 0))
$$

for all $\tau_{2} \in[0, T] \backslash \varepsilon_{0}$. At this point we are able to show that $u=\bar{u}$ almost everywhere if $u_{0}=\bar{u}_{0}$ almost everywhere. To this end we assume that $u_{0}=\bar{u}_{0}$ almost everywhere. Let now $p \in \Gamma_{0}$. We show that we find an open set $\tilde{U}_{p} \subset \Gamma_{0}$ containing $p$ such that $u=\bar{u}$ almost everywhere in $\bigcup_{t \in[0, \tilde{t}]} \Phi\left(\tilde{U}_{p}, t\right) \times\{t\}$ for some $\tilde{t}>0$. To this end let again $\psi$ be as in (9.4) with $\psi(\cdot, t): B_{R}(0) \rightarrow$ $\psi\left(B_{R}(0), t\right)$ where $B_{R}(0) \subset \mathbb{R}^{n}, \psi\left(B_{R}(0), t\right) \subset \Gamma(t)$ and $\psi(0,0)=p$. Furthermore we choose $0<r<R$ and set $\tilde{\psi}:=\psi(\cdot, 0)$ and $\tilde{U}:=\tilde{\psi}\left(B_{r}(0)\right) \subset \Gamma_{0}$. As in (9.35) we get a local Lipschitz constant $L$ of $f$ and have for $\tilde{t}:=\frac{r}{2 L}$ that

$$
\int_{S_{t}}|u(x, t)-\bar{u}(x, t)| \operatorname{det}(g(x, t)) \leqslant C \int_{B_{r}(0)}\left|u_{0}(x)-\bar{u}_{0}(x)\right| \operatorname{det}(g(x, 0))=0
$$

for all $t \in[0, \tilde{t}]$ and therefore $u=\bar{u}$ almost everywhere in $\bigcup_{t \in[0, \tilde{t}]} \Phi\left(\tilde{U}_{p}, t\right) \times\{t\}$ with $\tilde{U}_{p}:=$ $\tilde{\psi}\left(B_{\frac{r}{2}}(0)\right)$ since $S_{\tilde{t}}=B_{\frac{r}{2}}(0)$. Repeating the above argumentation for every $p \in \Gamma_{0}$ we get several sets $\tilde{U}_{p}$ whose union obviously covers $\Gamma_{0}$. By choosing a finite cover $\left\{\tilde{U}_{p_{i}}, i=1, \ldots, M\right\}$ we get several times $\tilde{t}_{i}$ such that $u=\bar{u}$ almost everywhere in $\bigcup_{t \in\left[0, \tilde{t}_{\min }\right]} \Gamma(t) \times\{t\}$ with $\tilde{t}_{\min }:=$ $\min _{i \in\{1, \ldots, M\}} \tilde{t}_{i}$. As $\tilde{t}_{\min }$ does not depend on time and because of (9.47) we can successively conclude that $u=\bar{u}$ almost everywhere in $G_{T}$.

The following Corollary follows immediately from the equivalence of Definition 4.3 and Definition 4.5 together with Theorem 9.4.

COROLlary 9.5 Let the assumptions of Theorem 9.4 be satisfied. Then the entropy solution of Definition 4.3 is unique. 


\section{Numerical algorithm}

Now we are going to derive a finite volume scheme for the initial value problem (1.1). Up to our knowledge the first finite volume scheme on evolving surfaces for parabolic equations was proposed by Lenz et al. [29]. They provide a scheme for diffusion on evolving surfaces. We adapt this scheme to nonlinear scalar conservation laws on evolving surfaces.

\section{$10.1 \quad$ Notation and preliminaries}

Following Dziuk and Elliot [14] the smooth initial surface $\Gamma_{0}$ is approximated by a triangulated surface $\Gamma_{0, h}$ which consists of a set of simplices (triangles for $n=2$ ) such that all its vertices $\left\{x_{j}^{0}\right\}_{j=1}^{N}$ sit on $\Gamma_{0}$. Such a set of simplices is called a triangulation $\mathcal{J}_{h}^{0}$ of $\Gamma_{0, h}$ and $h$ indicates the maximal diameter of a triangle on the whole family of triangulations. The triangulation $\mathcal{J}_{h}(t)$ and its $\Gamma(t)$ approximating surface $\Gamma_{h}(t)$ is defined by mapping the set of vertices $\left\{x_{j}^{0}\right\}_{j=1}^{N}$ with $\Phi(\cdot, t)$ onto $\Gamma(t)$, i.e.,

$$
x_{j}(t):=\Phi\left(x_{j}^{0}, t\right),
$$

i.e., they lie on motion trajectories. Thus, all the triangulations $\mathcal{J}_{h}(t)$ share the same grid topology. By this construction the set of simplices can be written as $\mathcal{J}_{h}(t)=\left\{T_{j}(t) \mid j=1, \ldots, M\right\}$ for $t \in[0, T]$, where $M$ is the time independent number of simplices. For the derivation of a finite volume scheme we introduce discrete time steps $t^{k}=k \tau$ where $\tau$ denotes the time step size and $k$ the time step index. For an arbitrary time step $t^{k}$ we have a smooth surface $\Gamma^{k}:=\Gamma\left(t^{k}\right)$, its approximation $\Gamma_{h}^{k}:=\Gamma_{h}\left(t^{k}\right)$ and the corresponding triangulation $\mathcal{J}_{h}^{k}:=\mathcal{J}_{h}\left(t^{k}\right)$ with simplices $T_{j}^{k}:=T_{j}\left(t^{k}\right)$. From [14] we know that for sufficiently small $h$ there is a uniquely defined lifting operator from $\Gamma_{h}^{k}$ onto $\Gamma^{k}$ via the orthogonal projection $\mathbb{P}^{k}=\mathbb{P}\left(t^{k}\right)$ in direction of the surface normal $v$ on $\Gamma^{k}$. For the comparison of quantities on $\Gamma^{k}$ and on $\Gamma_{h}^{k}$ we define curved simplices via the projection operator, i.e.

$$
\mathfrak{T}_{j}^{k}:=\mathbb{P}^{k} T_{j}^{k} .
$$

Such a projection $\mathfrak{T}_{j}^{k}$ propagates during the $(k+1)$ th time interval $\left[t^{k}, t^{k+1}\right]$. We define

$$
\mathfrak{T}_{j}^{k}(t):=\Phi\left(\left(\Phi\left(\cdot, t^{k}\right)\right)^{-1}\left(\mathfrak{T}_{j}^{k}\right), t\right)
$$

and denote by $V_{j}^{k}$ the $n$-dimensional measure of $T_{j}^{k}$.

\subsection{Definition of the finite volume scheme}

In order to derive a suitable finite volume scheme we integrate (1.1) over the curved simplex $\mathfrak{T}_{j}^{k}(t)$ and the time interval $\left[t^{k}, t^{k+1}\right]$. Applying the Leibniz formula and the Gauss theorem we obtain

$$
\begin{aligned}
0 & =\int_{t^{k}}^{t^{k+1}} \int_{\mathfrak{T}_{j}^{k}(t)} \dot{u}+u \nabla_{\Gamma_{t}} \cdot v+\nabla_{\Gamma_{t}} \cdot f(\cdot, \cdot, u) \\
& =\int_{t^{k}}^{t^{k+1}} \frac{d}{d t} \int_{\mathfrak{T}_{j}^{k}(t)} u+\int_{t^{k}}^{t^{k+1}} \int_{\partial \mathfrak{T}_{j}^{k}(t)} f(\cdot, \cdot, u) \cdot v_{\partial \mathfrak{T}_{j}^{k}(t)}
\end{aligned}
$$




$$
\begin{aligned}
& =\int_{\mathfrak{T}_{j}^{k}\left(t^{k+1}\right)} u-\int_{\mathfrak{T}_{j}^{k}\left(t^{k}\right)} u+\int_{t^{k}}^{t^{k+1}} \sum_{\mathfrak{e} \subset \partial \mathfrak{T}_{j}^{k}(t)} \int_{\mathfrak{e}} f(\cdot, \cdot, u) \cdot v_{\partial \mathfrak{T}_{j}^{k}(t)} \\
& =\int_{\mathfrak{T}_{j}^{k}\left(t^{k+1)}\right.} u-\int_{\mathfrak{T}_{j}^{k}} u+\int_{t^{k}} \sum_{\mathfrak{e} \subset \partial \mathfrak{T}_{j}^{k}(t)} \int_{\mathfrak{e}} f(\cdot, \cdot, u) \cdot v_{\partial \mathfrak{T}_{j}^{k}(t)},
\end{aligned}
$$

where $v_{\partial \mathfrak{T}_{j}^{k}(t)}$ denotes the unit outer normal along $\partial \mathfrak{T}_{j}^{k}(t)$ which is tangential to $\Gamma^{k}$ and $\mathfrak{e}$ denotes an edge of the boundary $\partial \mathfrak{T}_{j}^{k}(t)$. We introduce the approximations

$$
\int_{\mathfrak{T}_{j}^{k}\left(t^{k+1}\right)} u-\int_{\mathfrak{T}_{j}^{k}} u \approx V_{j}^{k+1} u_{j}^{k+1}-V_{j}^{k} u_{j}^{k}
$$

and

$$
\int_{t^{k}}^{t^{k+1}} \sum_{\mathfrak{e} \subset \partial \mathfrak{T}_{j}^{k}(t)} \int_{\mathfrak{e}} f(\cdot, \cdot, u) \cdot v_{\partial \mathfrak{T}_{j}^{k}(t)} \approx \tau \sum_{e \subset \partial T_{j}^{k}} g_{e, T_{j}^{k}}^{k}\left(u_{j}^{k}, u_{l(j, e)}^{k}\right),
$$

where $u_{j}^{k} \in \mathbb{R}$ represents the value of $u$ on $\mathfrak{T}_{j}^{k}, l(j, e)$ is the index of the simplex which shares the edge $e$ with $T_{j}^{k}$ and the $g_{e, \partial T_{j}^{k}}^{k}$ are some numerical flux functions which depend on cell number, edge and time index. We define the finite volume scheme by

AlGORITHM 10.1

$$
\begin{aligned}
u_{j}^{k+1} & :=\frac{1}{V_{j}^{k+1}}\left(V_{j}^{k} u_{j}^{k}-\tau \sum_{e \subset \partial T_{j}^{k}} g_{e, T_{j}^{k}}^{k}\left(u_{j}^{k}, u_{l(j, e)}^{k}\right)\right), \\
u_{j}^{0} & :=\frac{1}{V_{j}^{0}} \int_{\mathfrak{T}_{j}^{0}} u_{0} .
\end{aligned}
$$

For implementation purposes we introduce the approximated total mass on a cell $m_{j}^{k}:=V_{j}^{k} u_{j}^{k}$. In terms of these quantities the finite volume scheme (10.1) and (10.2) reads

$$
\begin{aligned}
m_{j}^{k+1} & :=m_{j}^{k}-\tau \sum_{e \subset \partial T_{j}^{k}} g_{e, T_{j}^{k}}^{k}\left(\frac{m_{j}^{k}}{V_{j}^{k}}, \frac{m_{l(j, e)}^{k}}{V_{l(j, e)}^{k}}\right), \\
m_{j}^{0} & :=\int_{\mathfrak{T}_{j}^{0}} u_{0} .
\end{aligned}
$$

EXAMPLE 10.2 (Engquist-Osher Numerical Flux) Using the notations from above we define for a simplex $T_{j}^{k} \in \mathcal{T}_{h}^{k}$ and an edge $e \subset \partial T_{j}^{k}$

$$
\begin{aligned}
& c_{e, T_{j}^{k}}^{k}(u):=\int_{e} f\left(\cdot, t^{k}, u\right) \cdot v_{\partial T_{j}^{k}}, \\
& c_{e, T_{j}^{k}}^{k,+}(u):=c_{e, T_{j}^{k}}^{k}(0)+\int_{0}^{u} \max \left\{c_{e, T_{j}^{k}}^{k}(s), 0\right\} d s, \\
& c_{e, T_{j}^{k}}^{k,-}(u):=\int_{0}^{u} \min \left\{c_{e, T_{j}^{k}}^{k}(s), 0\right\} d s .
\end{aligned}
$$


Then an Engquist-Osher-flux is defined by

$$
g_{e, T_{j}^{k}}^{k, \mathrm{EO}}(u, v):=c_{e, T_{j}^{k}}^{k,+}(u)+c_{e, T_{j}^{k}}^{k,-}(v) .
$$

\section{Numerical experiments}

The finite volume scheme (10.1) and (10.2) is validated by numerical experiments. To this end we formulate a linear transport problem on a sphere whose radius decreases exponentially in time. For this problem we know the exact solution and, thus, can compute some experimental orders of convergence (EOC). As a second test problem we choose a nonlinear (Burgers-like) flux function $f$ and state our problem on an ellipsoid which develops a narrowness in time.

Test Problem 1 (Linear)

We define

$$
\begin{aligned}
r(t) & :=\exp (-t), \\
\Gamma_{t} & :=\left\{r(t) x \mid x \in \mathbb{S}^{2}\right\}, \\
\Phi(x, t) & :=r(t) x, \\
\mathbf{V}(r(t) x) & :=\left(-x^{2}, x^{1}, 0\right)^{\mathrm{T}} \quad \text { for } x=\left(x^{1}, x^{2}, x^{3}\right) \in \mathbb{S}^{2}, \\
f(u) & :=2 \pi u, \\
f(r(t) x, t, u) & :=f(u) \mathbf{V}(r(t) x)
\end{aligned}
$$

and consider the initial value problem

$$
\begin{aligned}
\dot{u}+u \nabla_{\Gamma_{t}} \cdot v+\nabla_{\Gamma_{t}} \cdot f(\cdot, \cdot, u) & =0 \text { on } \quad G_{T}, \\
u(x, 0) & =u_{0}(x) \text { on } \Gamma_{0} .
\end{aligned}
$$

Since $v=\dot{r}(t) v$, where $v$ denotes the unit outer normal on $\mathbb{S}^{2}$, we have $\nabla_{\Gamma_{t}} \cdot v=\frac{2 \dot{r}(t)}{r(t)}=-2$ (cf. [14]). As in [30], one sees that the last term on the left hand side in (11.1) reads in polar coordinates $(r, \varphi, \theta)$

$$
\frac{1}{r(t)} \frac{\partial}{\partial \varphi} f(u)
$$

Thus (11.1) is equivalent to

$$
\dot{u}-2 u+\exp (t) \frac{\partial}{\partial \varphi} f(u)=0 \text { for } \varphi \in \mathbb{T}^{1}:=[0,2 \pi] \text { and } t>0,
$$

where $\varphi=0$ and $\varphi=2 \pi$ are identified. For given functions $\widetilde{u}_{0}: \mathbb{T}^{1} \rightarrow \mathbb{R}$ and $\widehat{u}:(0, \pi) \rightarrow \mathbb{R}$ we define in polar coordinates

$$
\begin{aligned}
\widetilde{u}(\varphi, t) & :=\exp (2 t) \widetilde{u}_{0}(\varphi-2 \pi(\exp (t)-1)), \\
u(\varphi, \theta, t) & :=\widetilde{u}(\varphi, t) \widehat{u}(\theta), \\
u_{0}(\varphi, \theta) & :=\widetilde{u}_{0}(\varphi) \widehat{u}(\theta) .
\end{aligned}
$$


TABLE 1. Experimental orders of convergence (EOC) for Test Problem 1, where $\bar{h}$ denotes the average diameter of the grid's elements.

\begin{tabular}{rccc}
\hline Elements & $\bar{h}$ & L1-Error & EOC \\
\hline 632 & 0.21605 & 1.86 & - \\
2,628 & 0.10613 & 1.53 & 0.27 \\
11,164 & 0.05145 & 1.16 & 0.39 \\
45,102 & 0.02557 & 0.76 & 0.59 \\
187,682 & 0.01251 & 0.49 & 0.61 \\
747,416 & 0.00627 & 0.30 & 0.68 \\
\hline
\end{tabular}

With these definitions one easily sees that $u$ is a solution of the initial value problem (11.1)(11.2). For testing our code we define

$$
\begin{aligned}
\widehat{u}(\theta) & :=\sin ^{2}(3 \theta) \mathbb{1}_{\{|\theta-\pi / 2|<\pi / 6\}}(\theta), \\
\widetilde{u}_{0}(\varphi) & :=\mathbb{1}_{\{\varphi<\pi\}}(\varphi)
\end{aligned}
$$

and choose an Engquist-Osher numerical flux. For our computations we use surface grids that approximate the sphere $\mathbb{S}^{2}$. They consist of flat triangles whose nodes lay on $\mathbb{S}^{2}$. We get the experimental orders of convergence which are listed in Table 1.

\section{Test Problem 2 (Nonlinear)}

The results of three further experiments are illustrated in Figures 1, 2 and 3, respectively. All three have the function

$$
u_{0}\left(x_{1}, x_{2}, x_{3}\right)=\cos ^{2}\left(\pi\left(x_{1}+2\right)\right) \mathbb{1}_{\left\{x_{1}<-3 / 2\right\}}\left(x_{1}\right)
$$

as initial values. For the first two experiments (see Figures 1 and 2) the flux function $f$ is constructed by taking a constant vector field which is pointing in direction of the $x_{1}$-axis and projecting it on the hypersurface $\Gamma_{t}$. This flux function is not divergence-free. Figure 1 shows the numerical solution of a Burgers equation on an evolving ellipsoid. You can see a shock that moves from left to right. In Figure 2 the same equation is considered, but due to fast change of geometry in the middle of the ellipsoid, the mass is compressed so fast that a second shock riding on the first one is induced. Thus, this second shock is induced by the change of geometry. For the third experiment (see Figure 3) the same parameters as in the second one are chosen, only the flux function is different, which is chosen to be divergence-free. Its construction is based on the following lemma which is a generalization of the one for the case of $\mathbb{S}^{2}$ developed by Ben-Artzi et al. [6].

LEMma 11.1 Given a function $h=h(x, t, u)$ which is defined for $t \in[0, T]$ and $u \in \mathbb{R}$ in a neighborhood of $\Gamma_{t}$, then the flux function defined by $f(x, t, u):=v(x, t) \times \nabla h(x, t, u)$ is divergence-free, where the $x$-dependence of $f$ is assumed to be of class $C^{2}$.

Proof. For fixed $t \in[0, T], u \in \mathbb{R}$ we consider a portion $\gamma(t)$ of $\Gamma_{t}$ with smooth boundary $\partial \gamma(t)$. Then by the divergence theorem we have

$$
\int_{\gamma(t)} \nabla_{\Gamma_{t}} \cdot f(x, t, u)=\int_{\partial \gamma(t)}(\mu(x, t) \times v(x, t)) \cdot \nabla h(x, t, u) .
$$



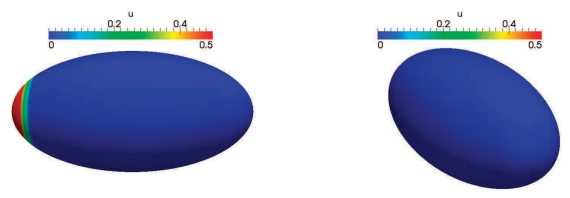

(a) $\mathrm{t}=0$
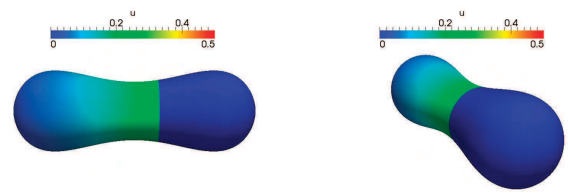

(c) $\mathrm{t}=0.4 \mathrm{~T}$


(e) $\mathrm{t}=0.8 \mathrm{~T}$
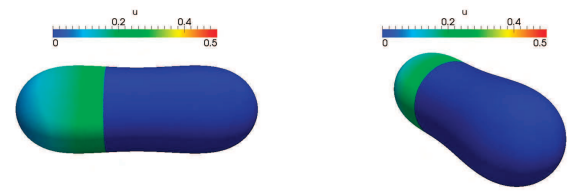

(b) $\mathrm{t}=0.2 \mathrm{~T}$
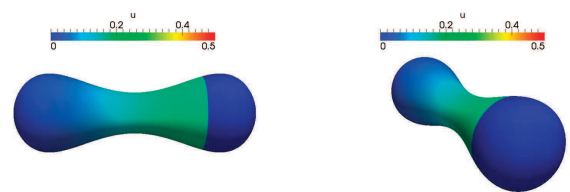

(d) $\mathrm{t}=0.6 \mathrm{~T}$
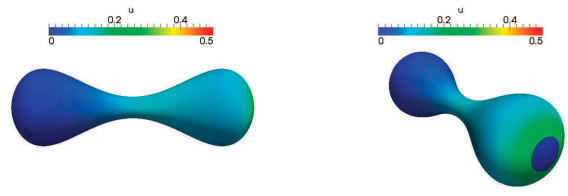

(f) $\mathrm{t}=\mathrm{T}$

FIG. 1. Burgers like shock on an evolving ellipsoid for several time steps. Here, $T$ denotes the end time.

As $\mu(x, t) \times v(x, t)$ is a unit tangent vector at $\partial \gamma(t)$ the integrand is the directional derivative along $\partial \gamma(t)$ und thus the integral vanishes for any smooth portion $\gamma(t)$.

For the third experiment a flux corresponding to $h(x, t, u)=-20 x_{3} u^{2}$ is chosen. The pictures from Figure 3 show the evolution of the numerical solution. Here, as in Figure 2 a second shock is geometrically induced and overtakes the first one.

\section{Appendix}

Proof of Lemma 2.6

Proof. It is sufficient to prove this relation locally. For this we use a parametrization $X=X(\theta, t)$,

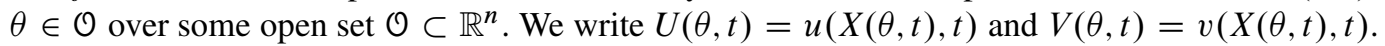
We use the notation $g_{i j}=X_{\theta_{i}} \cdot X_{\theta_{j}}$. The uppering of the indices has to be understood in the usual sense as inverting the matrix: $\left(g^{i j}\right)_{i, j=1, \ldots, n}=\left(g_{i j}\right)_{i, j=1, \ldots, n}^{-1}$.

Then, by definition of the material derivative (2.5) we have

$$
\begin{aligned}
\left(\underline{D}_{l} u\right)^{\circ} \circ X & =\frac{\partial}{\partial t}\left(\underline{D}_{l} u \circ X\right)=\frac{\partial}{\partial t}\left(g^{i j} U_{\theta_{j}} X_{\theta_{i}}^{l}\right) \\
& =\frac{\partial g^{i j}}{\partial t} U_{\theta_{j}} X_{\theta_{i}}^{l}+g^{i j} U_{t \theta_{j}} X_{\theta_{i}}^{l}+g^{i j} U_{\theta_{j}} X_{t \theta_{i}}^{l}
\end{aligned}
$$

We have that $U_{t}=\dot{u} \circ X$ and $X_{t}=V=v \circ X$. With the relation

$$
g_{t}^{i j}=-g^{i k} g^{j m}\left(V_{\theta_{k}} \cdot X_{\theta_{m}}+X_{\theta_{k}} \cdot V_{\theta_{m}}\right)
$$



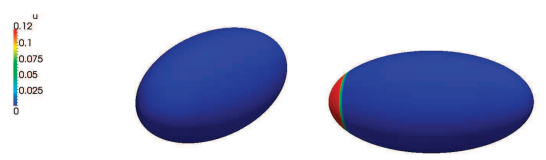

(a) $\mathrm{t}=0$


(c) $\mathrm{t}=0.50 \mathrm{~T}$

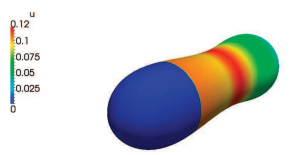

$\therefore$

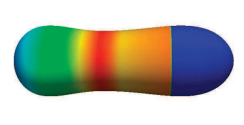

(e) $\mathrm{t}=0.58 \mathrm{~T}$


(g) $\mathrm{t}=0.67 \mathrm{~T}$
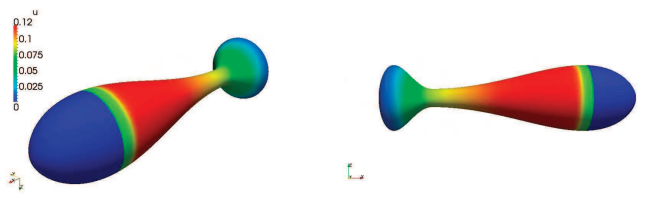

(i) $\mathrm{t}=0.91 \mathrm{~T}$

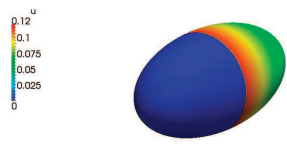

(b) $\mathrm{t}=0.40 \mathrm{~T}$

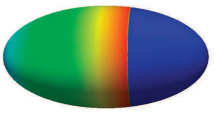

$\pm$

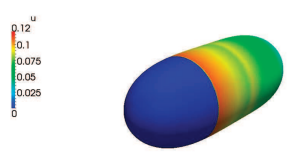

(d) $\mathrm{t}=0.54 \mathrm{~T}$

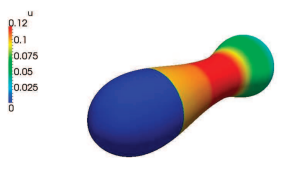

(f) $\mathrm{t}=0.62 \mathrm{~T}$

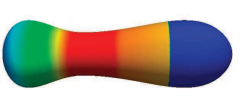

:

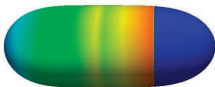

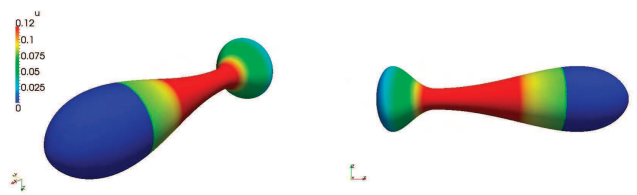

(h) $\mathrm{t}=0.73 \mathrm{~T}$

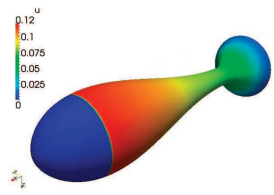

(j) $t=T$

FIG. 2. We see a Burgers like shock on an evolving ellipsoid. Caused by the deformation of the ellipsoid a second shock is produced and overtakes the first one. Here, $T$ denotes the end time.

we get

$$
\begin{aligned}
\left(\underline{D}_{l} u\right)^{\circ} \circ X & =-g^{i k} g^{j m}\left(V_{\theta_{k}}^{r} X_{\theta_{m}}^{r}+X_{\theta_{k}}^{s} V_{\theta_{m}}^{s}\right) U_{\theta_{j}} X_{\theta_{i}}^{l}+g^{i j} U_{t \theta_{j}} X_{\theta_{i}}^{l}+g^{i j} U_{\theta_{j}} X_{t \theta_{i}}^{l} \\
= & -g^{i k} V_{\theta_{k}}^{r} X_{\theta_{i}}^{l} g^{j m} X_{\theta_{m}}^{r} U_{\theta_{j}}-g^{i k} X_{\theta_{k}}^{s} X_{\theta_{i}}^{l} g^{j m} V_{\theta_{m}}^{s} U_{\theta_{j}}+g^{i j} U_{t \theta_{j}} X_{\theta_{i}}^{l}+g^{i j} U_{\theta_{j}} V_{\theta_{i}}^{l} \\
= & -\underline{D}_{l} u v_{r} \circ X \underline{D}_{r} u \circ X-\underline{D}_{s} x_{l} \circ X \nabla_{\Gamma} v_{s} \cdot \nabla_{\Gamma} u \circ X \\
& \quad+\underline{D}_{l} \dot{u} \circ X+\nabla_{\Gamma} u \cdot \nabla_{\Gamma} v_{l} \circ X .
\end{aligned}
$$



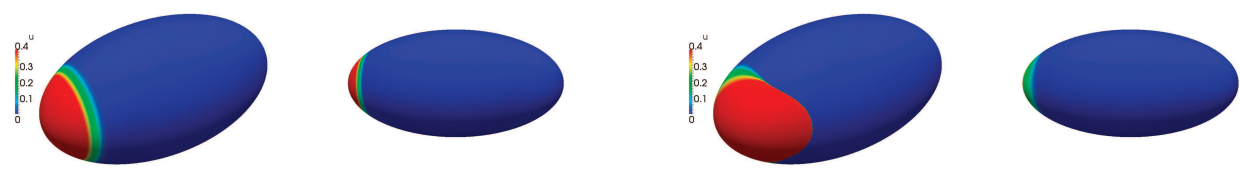

(a) $\mathrm{t}=0$

(b) $\mathrm{t}=0.02 \mathrm{~T}$
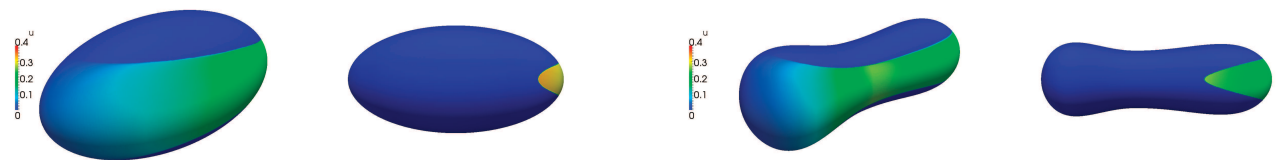

(c) $\mathrm{t}=0.22 \mathrm{~T}$

(d) $\mathrm{t}=0.26 \mathrm{~T}$
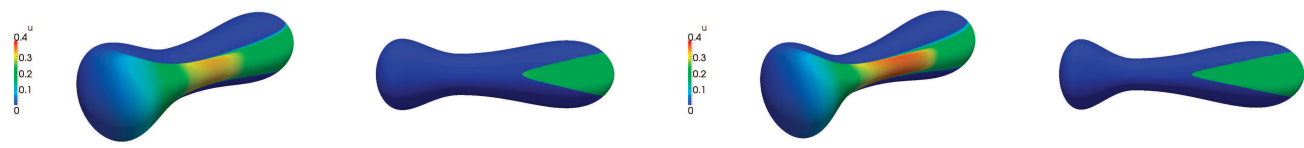

(e) $\mathrm{t}=0.28 \mathrm{~T}$

(f) $\mathrm{t}=0.29 \mathrm{~T}$


(g) $\mathrm{t}=0.33 \mathrm{~T}$

(h) $\mathrm{t}=0.40 \mathrm{~T}$
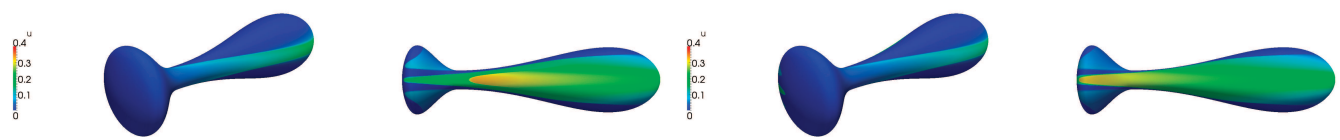

(i) $\mathrm{t}=0.58 \mathrm{~T}$

(j) $\mathrm{t}=0.64 \mathrm{~T}$
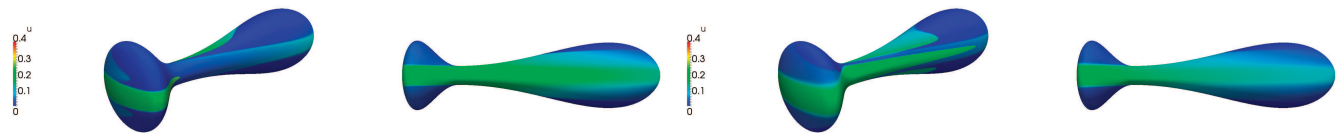

(k) $t=0.79 \mathrm{~T}$

(l) $\mathrm{t}=\mathrm{T}$

FIG. 3. As in Figure 2 a second shock is geometrically induced and overtakes the first one. Here, the flux function $f$ is divergence-free. $T$ denotes the end time.

Because of $\underline{D}_{s} x_{l}=P_{s l}=\delta_{s l}-v_{s} v_{l}$ we finally arrive at

$$
\begin{aligned}
\left(\underline{D}_{l} u\right)^{\cdot} & =\underline{D}_{l} \dot{u}-\underline{D}_{l} v_{r} \underline{D}_{r} u-P_{s l} \underline{D}_{r} v_{s} \underline{D}_{r} u+\underline{D}_{r} u \underline{D}_{r} v_{l} \\
& =\underline{D}_{l} \dot{u}-\underline{D}_{r} u\left(\underline{D}_{l} v_{r}+P_{s l} \underline{D}_{r} v_{s}-\underline{D}_{r} v_{l}\right) .
\end{aligned}
$$

The Lemma is proved. 


\section{Acknowledgments}

The work has been supported by Deutsche Forschungsgemeinschaft via SFB/TR 71 'Geometric Partial Differential Equations'.

\section{REFERENCES}

1. Amorim, P., Ben-Artzi, M. \& LeFloch, P. G., Hyperbolic conservation laws on manifolds: total variation estimates and the finite volume method. Methods Appl. Anal. 12 (2005), 291-323. Zbl1114. 35121 MR2254012

2. Amorim, P., LeFloch, P. G. \& Neves, W., A geometric approach to error estimates for conservation laws posed on a spacetime. Nonlinear Anal. 74 (2011), 4898-4917. Zbl1221. 35229 MR2810675

3. Aubin, T., Nonlinear analysis on manifolds: Monge-Ampère equations. Reihe Grundlehren der Mathematischen Wissenschaften [Fundamental Principles of Mathematical Sciences] 252 (1982). Zbl0512.53044 MR0681859

4. BALE, D. S., Wave propagation algorithms on curved manifolds with applications to relativistic hydrodynamics. Thesis. MR2703081

5. Berger, M. J., Calhoun, D. A., Helzel, C. \& LeVeque, R. J., Logically rectangular finite volume methods with adaptive refinement on the sphere. Philos. Trans. R. Soc. Lond. Ser. A Math. Phys. Eng. Sci. 367 (2009), 4483-4496. Zbl1192.76028 MR2550979

6. Ben-Artzi, M., Falcovitz, J. \& LeFloch, P. G., Hyperbolic conservation laws on the sphere: A geometry-compatible finite volume scheme. J. Comput. Phys. 228 (2009), 5650-5668. Zbl1168.65372 MR2542909

7. Ben-Artzi, M. \& LeFloch, P. G., Well-posedness theory for geometry-compatible hyperbolic conservation laws on manifolds. Ann. Inst. Henri Poincaré, Anal. Non Linéaire 24 (2007), 989-1008. Zbl1138.35055 MR2371116

8. Calhoun, D. A., Helzel, C. \& LeVeque R. J., Logically rectangular grids and finite volume methods for PDEs in circular and spherical domains. SIAM Review 50 (2008), 723-752. Zbl1155. 65061 MR2460802

9. Cermelli, P., Fried, E. \& Gurtin, M. E., Transport relations for surface integrals arising in the formulation of balance laws for evolving fluid interfaces. J. Fluid Mech. 544 (2005), 339-351. Zbl1083. 76061 MR2262061

10. Chen, G. Q. \& FRID, H., Divergence-measure fields and hyperbolic conservation laws. Arch. Ration. Mech. Anal. 147 (1999), 89-118. Zbl0942.35111 MR1702637

11. Chen, G. Q. \& RASCle, M., Initial layers and uniqueness of weak entropy solutions to hyperbolic conservation laws. Arch. Ration. Mech. Anal. 153 (2000), 205-220. Zb10962.35122 MR1771520

12. Dafermos, C. M., Hyperbolic conservation laws in continuum physics. Grundlehren der Mathematischen Wissenschaften [Fundamental Principles of Mathematical Sciences] 325 (2010). Zbl1196.35001 MR2574377

13. Deckelnick, K., Dziuk, G. \& Elliott, C. M., Computation of geometric partial differential equations and mean curvature flow. Acta Numerica 14 (2005), 139-232. Zbl1113.65097

14. Dziuk, G. \& Elliott, C., Finite elements on evolving surfaces. IMA J. Numer. Anal. 27 (2007), 262-292. Zbl1120.65102 MR2317005

15. Dziuk, G. \& Elliott, C., L2-estimates for the evolving surface finite element method. Math. Comp. 82 (2013), 1-24. Zblpre06147261 MR2983013

16. Dziuk, G. \& Elliott, C., A Fully discrete evolving surface finite element method. SIAM J. Numer. Anal. 50 (2012), 2677-2694. Zblpre06128716 MR3022237 
17. Dziuk, G., Lubich, C. \& Mansour, D., Runge-Kutta time discretization of parabolic differential equations on evolving surfaces. IMA J. Numer. Anal. 32 (2012), 394-416. Zb11247.65124 MR2911394

18. Giesselmann, J. \& Wiebe, M., Finite volume schemes for balance laws on time-dependent surfaces. In: Numerical Methods for Hyperbolic Equations, CRC Press, London, (2012), 251-258.

19. Gilbarg, D. \& Trudinger, N. S., Elliptic partial differential equations of second order. Springer Berlin Heidelberg (2001). Zbl1042 . 35002 MR1814364

20. Hermsdörfer, K., Kraus, C. \& Kröner, D., Interface conditions for limits of the Navier-StokesKorteweg modell. Interfaces Free Bound. 13 (2011), 239-254. Zb11219. 35191 MR2813525

21. JUNG, N., HAASDONK, B. \& KRÖNER, D., Reduced basis method for quadratically nonlinear transport equations. Int. J. Computing Science \& Mathematics 2 (2009), 334-353. Zbl1189.65225 MR2606093

22. KRÖNER, D. \& MÜller, T., Related problems for TV-estimates for conservation laws on surfaces. In: Tatsien Li; Song Jiang (eds.) Hyperbolic Problems: Theory, Numerics and Applications, Ser. Contemp. Appl. Math. CAM 18 (2012), 584-592.

23. KRÖNER, D., Jump conditions across phase boundaries for the Navier-Stokes Korteweg system. In: Tatsien Li; Song Jiang (eds.) Hyperbolic Problems: Theory, Numerics and Applications, Ser. Contemp. Appl. Math. CAM 18 (2012), 494-501.

24. KRÖNER, D., Numerical schemes for conservation laws. Wiley-Teubner Series Advances in Numerical Mathematics. John Wiley \& Sons Ltd., 1997. Zb10872.76001 MR1437144

25. KRUŽKOv, S. N., First order quasilinear equations with several independent variables. Mat. Sb. (N.S.) 81 (1970), 228-255. MR0267257

26. LeFloch, P. G. \& Stewart, J. M., Shock waves and gravitational waves inmatter spacetimes with Gowdy symmetry. Portugalieae Mathematica 62 (2005), Fasc. 3, Nova Serie.

27. LeFloch, P. G., Neves, W. \& OKutmustur, B., Hyperbolic conservation laws on manifolds. Error estimate for finite volume schemes. Acta Math. Sin., Engl. Ser. 25 (2009), 1041-1066. Zb11178. 35251

28. Lengeler, D. \& Müller, T., Scalar conservation laws on constant and time-dependent Riemannian manifolds. J. Differential Equations 254 (2013), 1705-1727. Zbl pre06140128 MR3003289

29. Lenz, M., Nemadjieu, S. F. \& Rumpf, M., A convergent finite volume scheme for diffusion on evolving surfaces. SIAM J. Numer. Anal. 49 (2011), 15-37. Zbl1254. 65095 MR2764419

30. MÜlLER, T., Erhaltungsgleichungen auf Mannigfaltigkeiten Wohlgestelltheit, Totalvariationsabschätzungen und Numerik. Diplomarbeit, Freiburg (2010).

31. Rossmanith, J. A., A wave propagation method with constrained transport for ideal and shallow water magnetohydrodynamics. Thesis. MR2703746

32. Rossmanith, J. A, Bale, D. S. \& LeVeque. R. J., A wave propagation algorithm for hyperbolic systems on curved manifolds. J. Comput. Phys. 199 (2004), 631-662. Zb11126. 76350 Mr2091909

33. PAnov, E. Y., On the Cauchy problem for a first-order quasilinear equation on a manifold. Differential Equations 33 (1997), 257-266. Zb10908.58071 MR1609868 